This document is the accepted manuscript version of the following article:

Dietzel, A., \& Reichert, P. (2012). Calibration of computationally demanding and structurally uncertain models with an application to a lake water quality model. Environmental Modelling and Software, 38, 129-146. http:// doi.org/10.1016/j.envsoft.2012.05.007

This manuscript version is made available under the CC-BY-NC-ND 4.0

license http://creativecommons.org/licenses/by-nc-nd/4.0/

\title{
Calibration of computationally demanding and structurally uncertain models with an application to a lake water quality model
}

\author{
Anne Dietzel ${ }^{\mathrm{a}, \mathrm{b}}$ and Peter Reichert ${ }^{\mathrm{a}, \mathrm{b}}$ \\ ${ }^{a}$ Eawag, Swiss Federal Institute of Aquatic Science and Technology, 8600 Dübendorf, \\ Switzerland \\ b Institute of Biogeochemistry and Pollutant Dynamics, ETH Zurich, 8092 Zurich, Switzerland
}

\begin{abstract}
Models of environmental systems are simplified representations of the reality. For this reason, their results are affected by systematic errors. This bias makes it difficult to get reliable uncertainty estimates of model parameters and predictions. A relatively simple way of considering this bias when using deterministic models is to add a statistical representation of the bias to the model output in addition to observation error and to jointly estimate model parameters, bias and observation error. When assuming Normal distributions for bias and observation error, this leads to a relatively simple likelihood function that can easily be evaluated. Nevertheless, the sampling from the posterior distribution still requires long Markov chains to be calculated which can be prohibitive for computationally demanding models. In order to extend the range of applicability of this technique to computationally demanding models, we suggest to replace Markov chain sampling by a Normal approximation to the posterior of the parameters and to estimate prediction uncertainty by linearized error propagation. We tested this procedure for a didactical example and for an application of the biogeochemical-ecological lake model BELAMO to long-term data from Lake Zurich. This is a good test application because the strong coupling of output variables makes it difficult to avoid bias in the results of this model. These tests demonstrate the applicability of the suggested procedure, the approximate reproduction of the results of the full procedure for the didactical example, and meaningful results for the lake model. For the latter, the results demonstrate that the assumption of a realistic likelihood function leads to the conclusion that prediction uncertainty may be high.
\end{abstract}

Keywords: Bias; Lake water quality model; Structurally uncertain model; Model complexity; Multiobjective calibration.

\section{Pre-published version;}

The final version is published in:

Environmental Modelling \& Software, 38, 129-146. DOI: 10.1016/j.envsoft.2012.05.007 


\section{Introduction}

Models substantially contribute to formalizing and summarizing knowledge, analyzing observations and testing hypotheses about the structure and function of environmental systems. As all models are simplified representations of reality, results of environmental models are, in addition to input and parametric uncertainty, affected by errors in model structure. All of these elements lead to systematic deviations of model results from observations. Such systematic deviations, also called model inadequacy or bias, are defined as deviations of the mean of the real output of the system from the predictions of the expected value of the model (Kennedy and O'Hagan 2001). The presence of bias is ubiquitous in environmental modeling and, if it can be assumed to be constant in time or described by a functional dependence on influence factors, predictions can be corrected based on the identification of bias in the past (Dee and Da Silva 1998, Christensen et al. 2008, Buser et al. 2009). However, in many cases it may not be possible to extrapolate bias easily as it may be time-dependent and does not show a simple dependence on external influence factors or model variables. As long as model bias is not too large, the model might still reproduce key output patterns of the underlying system and the environmental scientist may be content with its explanatory power (being aware that no model will be perfect). However, when trying to identify model parameters from data by using statistical inference and when using the model for probabilistic prediction, model bias leads to a violation of typical statistical assumptions, such as randomly and independently distributed observations around the mean model prediction. This leads to unreliable uncertainty estimates of model parameters and predictions. As the definition of bias is a frequentist concept, it can either be identified by repeated experiments or by residual analysis e.g. of time series calculated at the best estimates of the true parameter values.

Different ways have been suggested to cope with this problem. Reducing model bias by improving the deterministic model, i.e. describing the mean system behavior more realistically, is the most obvious solution. However, it may also be necessary to account for elements of system dynamics not covered by the deterministic model by making the model stochastic (Vrugt et al. 2005, Vrugt and Robinson 2007; Lin and Beck 2007; Reichert and Mieleitner 2009, Bulygina and Gupta 2011). Both solutions result in a higher model complexity and can increase the computational burden considerably. As a different, computationally cheaper, option to derive reliable uncertainty bounds of model predictions, it was suggested to use a statistical description of bias in model output based on a Bayesian framework Craig et al. 1996, Craig et al. 2001, Kennedy and O'Hagan 2001, Higdon et al. 2004, Bayarri et al. 2007).

In addition to unreliable uncertainty bounds, another problem resulting from systematic deviations between model results and data is that frequently used model parameter estimation techniques often cause results that do not fulfill calibration objectives of the modeler. A scientist calibrating a model 
manually may weigh different calibration objectives, e.g. the peaks and the recessions of a hydrograph, differently. To address this problem, manual calibration criteria were included explicitly by using multipbjective optimization techniques for model calibration (Yapo et al. 1998, Gupta et al. 1998, Madsen 2000: Madsen et al. 2002 Gupta et al. 2003 Boyle et al. 2003, Savić et al. 2011). The resulting "Pareto set" summarizes all combinations of parameter values for which improving one calibration criterion by changing the parameter values is only possible by worsening another criterion. It thus provides an overview of ways of considering the different calibration criteria without making a unique choice of their weights. For this reason it is typical that the Pareto set contains solutions that are more satisfying to the modeler than the solution resulting from a single-objective optimization technique that does not explicitly weigh between different calibration objectives. On the other hand, the Pareto set does not provide probabilistic information required for estimating prediction uncertainty.

To assess the parameter, structural and prediction uncertainty under the problems mentioned above, a combination of statistical description of model bias Kennedy and O'Hagan 2001 Bayarri et al. 2007) and the ideas underlying multi-objective model calibration (Yapo et al. 1998, Gupta et al. 1998; Gupta et al. 2003, Boyle et al. 2003) was suggested by Reichert and Schuwirth (2012) System observations are described as the sum of deterministic model output, bias and measurement error. The measurement error is assumed to be uncorrelated and normally distributed, whereas our prior knowledge of the bias is represented by a Gaussian stochastic process. The identifiability problem between model and bias was addressed by specifying a prior for the bias which favors narrow distributions and has a mean of zero. This intends to support the description of the main output patterns by the model rather than by the bias. Identifiability can further be improved by an informative prior of the observation error about which typically more knowledge is available than about the bias. The prior of the statistical description of bias was used to weigh between different calibration objectives. The modeler can choose how much bias he or she is willing to accept in different model output variables. This approach introduces subjective elements into the calibration procedure; but this seems unavoidable in the presence of bias as there is no fundamental criterion that would specify how to "distribute" bias between different model variables. As the modeler can specify different priors for bias in different variables, or even different priors for different parts of a time series of a single variable, this procedure obviously links statistical bias description to multi-objective calibration. The result will not be the full Pareto set corresponding to different calibration objectives, but a posterior distribution which considers the modeler's weights of different criteria. This posterior distribution can then be used to derive probabilistic uncertainty bounds of model predictions.

The technique as described by Reichert and Schuwirth (2012) is computationally much cheaper than most of the techniques involving stochastic models (Vrugt et al. 2005. Vrugt and Robinson 2007 Lin and Beck 2007; Reichert and Mieleitner 2009: Bulygina and Gupta 2011) but it still requires tens 
of thousands of simulation runs to get a Markov chain sample of the posterior. For computationally demanding simulation programs, this may still be prohibitive. As it is important to extend the range of applicability of this calibration and prediction technique to computationally more demanding models, it is the goal of this paper to derive computationally efficient methods for the approximation of the posterior. The basic idea is to replace Markov chain sampling of the posterior by a Normal distribution that approximates the posterior at its maximum and to use linearized error propagation to derive approximate uncertainty bounds of model predictions. Those approximations do not rely on the specific formulation of the likelihood function used herein, but could be used in other contexts where an approximation of the posterior distribution by a normal distribution seems to be meaningful and where a full Markov chain sampling is computationally too expensive. They are specifically useful to apply the technique described by Reichert and Schuwirth (2012) to computationally demanding environmental models. To test this procedure, we applied it to the same didactical example as used by Reichert and Schuwirth (2012) and to long-term simulations of the lake model BELAMO.

This paper is structured as follows: In section 2 we briefly review the key equations of the technique as described by Reichert and Schuwirth (2012) and then derive the new approximation for its numerical implementation. The following two sections 3 and 4 are devoted to a didactical example and the application of the new technique to long-term simulations of the lake model BELAMO. Finally, in section 5 we draw our conclusions.

\section{Methods}

We briefly summarize the technique suggested by Reichert and Schuwirth (2012) that combine statistical bias description with ideas of multi-objective model calibration and then discuss options to simplify the numerical implementation of this approach to get an approximate technique that is computationally less demanding.

\subsection{Review of Inference in the Presence of Bias}

\subsubsection{Likelihood Function}

As mentioned in the introduction, in the presence of bias, the mean of the true model outcomes is no longer identical to the expected value of the model prediction. This means that the description of the observations as a sum of a deterministic function describing the mean, $\mathbf{y}_{M}^{L}(\mathbf{x}, \boldsymbol{\theta})$, and an observation error with mean zero, $\mathbf{E}^{L}(\boldsymbol{\psi})$,

$$
\mathbf{Y}_{M}^{L}(\mathbf{x}, \boldsymbol{\theta}, \boldsymbol{\psi})=\mathbf{y}_{M}^{L}(\mathbf{x}, \boldsymbol{\theta})+\mathbf{E}^{L}(\boldsymbol{\psi})
$$


is no longer valid. In this equation, the vector $\mathbf{Y}_{M}^{L}$ of random variables represents the observations as described by the model $M$ at the observation layout $L$ that defines the output variables and the time points and locations at which they are observed or evaluated, $\mathbf{y}_{M}^{L}(\mathbf{x}, \boldsymbol{\theta})$ is a parameterization of the mean model response as a function of inputs, $\mathbf{x}$ and model parameters, $\boldsymbol{\theta}$, and $\mathbf{E}^{L}(\boldsymbol{\psi})$ is a vector of random variables that represents the observation error, which is assumed to have zero mean and may depend on additional parameters, $\psi$. Defining the bias as the difference of the mean of the observations and the expected value of the model

$$
\mathbf{b}=\mathrm{E}\left[\mathbf{Y}_{M}^{L}(\mathbf{x}, \boldsymbol{\theta}, \boldsymbol{\psi})\right]-\mathbf{y}_{M}^{L}(\mathbf{x}, \boldsymbol{\theta})
$$

we end with the modified description of the observations by

$$
\mathbf{Y}_{M}^{L}(\mathbf{x}, \boldsymbol{\theta}, \boldsymbol{\psi}, \boldsymbol{\xi})=\mathbf{y}_{M}^{L}(\mathbf{x}, \boldsymbol{\theta})+\mathbf{b}_{M}^{L}(\mathbf{x}, \boldsymbol{\xi})+\mathbf{E}^{L}(\boldsymbol{\psi})
$$

where $\mathbf{b}_{M}^{L}(\mathbf{x}, \boldsymbol{\xi})$ is now an unknown deterministic function of the input, $\mathbf{x}$, and additional parameters, $\boldsymbol{\xi}$, describing the bias. Note that this bias term combines bias in the model with (potential) bias in the observation process.

The application of equation (3) to observed data leads to a significant identifiability problem between model and bias, as we can only get information on the sum of the two. Following the diterature on statistical bias description, Craig et al. 1996. Craig et al. 2001 Kennedy and O'Hagan 2001; Higdon et al. 2004; Bayarri et al. 2007), we solve this problem by describing our knowledge of the bias by a probability distribution in the Bayesian sense. This makes the bias a random variable, $\mathbf{B}_{M}^{L}(\mathbf{x}, \boldsymbol{\xi})$, and leads to the following description of our knowledge of new observations:

$$
\mathbf{Y}_{M}^{L}(\mathbf{x}, \boldsymbol{\theta}, \boldsymbol{\psi}, \boldsymbol{\xi})=\mathbf{y}_{M}^{L}(\mathbf{x}, \boldsymbol{\theta})+\mathbf{B}_{M}^{L}(\mathbf{x}, \boldsymbol{\xi})+\mathbf{E}^{L}(\boldsymbol{\psi})
$$

Equation (4) leads to a hierarchical model with the bias, $\mathbf{B}_{M}^{L}(\mathbf{x}, \boldsymbol{\xi})$, as an intermediate variable. Integrating out this intermediate variable, a likelihood function can be obtained as a function of the parameters, only:

$$
f_{\mathbf{Y}_{M}^{L} \mid \boldsymbol{\Theta}, \boldsymbol{\Psi}, \boldsymbol{\Xi}}\left(\mathbf{y}^{L} \mid \boldsymbol{\theta}, \boldsymbol{\psi}, \boldsymbol{\xi}, \mathbf{x}\right)=\int f_{\mathbf{E}^{L} \mid \Psi}\left(\mathbf{y}^{L}-\mathbf{y}_{M}^{L}(\mathbf{x}, \boldsymbol{\theta})-\mathbf{b}^{L} \mid \boldsymbol{\psi}\right) \cdot f_{\mathbf{B}_{M}^{L} \mid \boldsymbol{\Xi}}\left(\mathbf{b}^{L} \mid \boldsymbol{\xi}, \mathbf{x}\right) \mathrm{d} \mathbf{b}^{L}
$$

In this equation, $f_{\mathbf{E}^{L} \mid \Psi}$ is the probability density of the observation error and $f_{\mathbf{B}_{M}^{L} \mid \Xi}$ represents the prior density of the bias given the parameters $\boldsymbol{\xi}$ and external inputs $\mathbf{x}$.

We assume normally distributed observation errors with mean zero and covariance matrix $\boldsymbol{\Sigma}_{\mathbf{E}^{L}}$ 
$\left(n_{L}\right.$ is the number of observations in layout $\left.L\right)$

$$
f_{\mathbf{E}^{L} \mid \boldsymbol{\Psi}}\left(\boldsymbol{\epsilon}^{L} \mid \boldsymbol{\psi}\right)=\frac{1}{\sqrt{2 \pi}^{n}} \frac{1}{\sqrt{\operatorname{det}\left(\boldsymbol{\Sigma}_{\mathbf{E}^{L}}(\boldsymbol{\psi})\right)}} \exp \left(-\frac{1}{2}\left(\boldsymbol{\epsilon}^{L}\right)^{\mathrm{T}} \boldsymbol{\Sigma}_{\mathbf{E}^{L}}(\boldsymbol{\psi})^{-1} \boldsymbol{\epsilon}^{L}\right)
$$

and a Gaussian stochastic process with mean zero and covariance matrix $\boldsymbol{\Sigma}_{\mathbf{B}_{M}^{L}}$ for the bias

$$
f_{\mathbf{B}_{M}^{L} \mid \boldsymbol{\Xi}}\left(\mathbf{b}^{L} \mid \boldsymbol{\xi}, \mathbf{x}\right)=\frac{1}{\sqrt{2 \pi}^{n_{L}}} \frac{1}{\sqrt{\operatorname{det}\left(\boldsymbol{\Sigma}_{\mathbf{B}_{M}^{L}}(\boldsymbol{\xi}, \mathbf{x})\right)}} \exp \left(-\frac{1}{2}\left(\mathbf{b}^{L}\right)^{\mathrm{T}} \boldsymbol{\Sigma}_{\mathbf{B}_{M}^{L}}(\boldsymbol{\xi}, \mathbf{x})^{-1} \mathbf{b}^{L}\right)
$$

The choice for a Gaussian stochastic process is motivated by the fact that this is an easy formulation of our knowledge of the bias including a correlation structure and only a small number of parameters (Bayarri et al. 2007). This formulation makes it possible to separate the errors into a random observation error and a systematic component representing the bias (earlier approaches with autoregressive error models already considered the dependence structure of the residuals but did not make the attempt to separate observation error and bias (Kuczera 1983, Bates and Campbell 2001; Yang et al. 2007)). Under these normality assumptions we can do the integration in (5) analytically and obtain the likelihood function

$$
\begin{aligned}
f_{\mathbf{Y}_{M}^{L} \mid \boldsymbol{\Theta}, \boldsymbol{\Psi}, \boldsymbol{\Xi}}\left(\mathbf{y}^{L} \mid \boldsymbol{\theta}, \boldsymbol{\psi}, \boldsymbol{\xi}, \mathbf{x}\right)= & \frac{1}{\sqrt{2 \pi}^{n_{L}}} \frac{1}{\sqrt{\operatorname{det}\left(\boldsymbol{\Sigma}_{\mathbf{E}^{L}}+\boldsymbol{\Sigma}_{\left.\mathbf{B}_{M}^{L}\right)}\right.}} \\
& \cdot \exp \left(-\frac{1}{2}\left[\mathbf{y}^{L}-\mathbf{y}_{M}^{L}(\mathbf{x}, \boldsymbol{\theta})\right]^{\mathrm{T}}\left(\boldsymbol{\Sigma}_{\mathbf{E}^{L}}+\boldsymbol{\Sigma}_{\mathbf{B}_{M}^{L}}\right)^{-1}\left[\mathbf{y}^{L}-\mathbf{y}_{M}^{L}(\mathbf{x}, \boldsymbol{\theta})\right]\right) .
\end{aligned}
$$

The simplest assumptions of the correlation structures of the covariance matrices in (8) would be to assume independence between the different components of the observation error, $\boldsymbol{\Sigma}_{\mathbf{E}^{L}}$, and correlation coefficients that decrease with increasing distance of the independent input variables, $\mathbf{x}$, for the bias, $\boldsymbol{\Sigma}_{\mathbf{B}_{M}^{L}}$. This leads to the expressions:

$$
\Sigma_{\mathbf{E}^{L}, i, j}(\boldsymbol{\psi})=\left\{\begin{array}{lc}
\sigma_{\mathbf{E}^{L}, i}(\boldsymbol{\psi})^{2} & \text { for } i=j \\
0 & \text { else }
\end{array}\right.
$$

and

$$
\Sigma_{\mathbf{B}_{M}^{L}, i, j}(\boldsymbol{\xi}, \mathbf{x})= \begin{cases}0 & \text { if } y_{i}^{L} \text { and } y_{j}^{L} \text { are of different type } \\ \sigma_{\mathbf{B}_{M}^{L}, i}(\boldsymbol{\xi}) \sigma_{\mathbf{B}_{M}^{L}, j}(\boldsymbol{\xi}) \exp \left(-\sum_{k} \beta_{k}(\boldsymbol{\xi})\left(x_{i, k}-x_{j, k}\right)^{2}\right) & \text { else }\end{cases}
$$

The smoothing parameter $\beta_{k}$ for input dimension $k$ (e.g. time or space) in this equation relate to the corresponding correlation lengths, $\tau_{k}=1 / \sqrt{\beta_{k}}$. Equation (10) assumes no correlation between output variables of different type, e.g. concentrations of different substances. This assumption, as well as the 
specific form of equation 10 is not crucial for the procedure but will be used in our applications.

\subsubsection{Inference}

We can infer parameter values from the posterior density that is proportional to the prior $f_{\boldsymbol{\Theta}, \boldsymbol{\Psi}, \boldsymbol{\Xi}}$, of the parameters times the likelihood function (5). With observations, $\mathbf{y}^{L_{1}}$, at points in time and/or space described by the observation layout $L_{1}$, we obtain:

$$
f_{\boldsymbol{\Theta}, \boldsymbol{\Psi}, \boldsymbol{\Xi} \mid \mathbf{Y}_{M}^{L_{1}}}\left(\boldsymbol{\theta}, \boldsymbol{\psi}, \boldsymbol{\xi} \mid \mathbf{y}^{L_{1}}, \mathbf{x}\right) \propto f_{\boldsymbol{\Theta}, \boldsymbol{\Psi}, \boldsymbol{\Xi}}(\boldsymbol{\theta}, \boldsymbol{\psi}, \boldsymbol{\xi}) \cdot f_{\mathbf{Y}_{M}^{L_{1} \mid \boldsymbol{\Theta}, \boldsymbol{\Psi}, \boldsymbol{\Xi}}}\left(\mathbf{y}^{L_{1}} \mid \boldsymbol{\theta}, \boldsymbol{\psi}, \boldsymbol{\xi}, \mathbf{x}\right)
$$

Using the Normal distributions (6) and (7) and the resulting likelihood function (8), we get:

$$
\begin{aligned}
f_{\boldsymbol{\Theta}, \boldsymbol{\Psi}, \boldsymbol{\Xi} \mid \mathbf{Y}_{M}^{L_{1}}}\left(\boldsymbol{\theta}, \boldsymbol{\psi}, \boldsymbol{\xi} \mid \mathbf{y}^{L_{1}}, \mathbf{x}\right) \propto \frac{f_{\boldsymbol{\Theta}, \boldsymbol{\Psi}, \boldsymbol{\Xi}}(\boldsymbol{\theta}, \boldsymbol{\psi}, \boldsymbol{\xi})}{\sqrt{\operatorname{det}\left(\boldsymbol{\Sigma}_{\mathbf{E}^{L_{1}}}+\boldsymbol{\Sigma}_{\left.\mathbf{B}_{M}^{L_{1}}\right)}\right.}} \\
\cdot \exp \left(-\frac{1}{2}\left[\mathbf{y}^{L_{1}}-\mathbf{y}_{M}^{L_{1}}(\mathbf{x}, \boldsymbol{\theta})\right]^{\mathrm{T}}\left(\boldsymbol{\Sigma}_{\mathbf{E}^{L_{1}}}+\boldsymbol{\Sigma}_{\mathbf{B}_{M}^{L_{1}}}\right)^{-1}\left[\mathbf{y}^{L_{1}}-\mathbf{y}_{M}^{L_{1}}(\mathbf{x}, \boldsymbol{\theta})\right]\right) .
\end{aligned}
$$

(note that $\boldsymbol{\Sigma}_{\mathbf{E}^{L_{1}}}$ depends on $\boldsymbol{\psi}$ and $\boldsymbol{\Sigma}_{\mathbf{B}_{M}^{L_{1}}}$ on $\boldsymbol{\xi}$ ). To get information about the bias, we can calculate the conditional distribution of the bias given the observations and parameters, which is proportional to the joint distribution of bias and observed outputs:

$$
\begin{aligned}
f_{\mathbf{B}_{M}^{L_{1}} \mid \mathbf{Y}_{M}^{L_{1}}, \boldsymbol{\Theta}, \boldsymbol{\Psi}, \boldsymbol{\Xi}}\left(\mathbf{b}^{L_{1}} \mid \mathbf{y}^{L_{1}}, \boldsymbol{\theta}, \boldsymbol{\psi}, \boldsymbol{\xi}, \mathbf{x}\right) & \propto f_{\mathbf{Y}_{M}^{L_{1}, \mathbf{B}_{M}^{L_{1}} \mid \boldsymbol{\Theta}, \boldsymbol{\Psi}, \boldsymbol{\Xi}}}\left(\mathbf{y}^{L_{1}}, \mathbf{b}^{L_{1}} \mid \boldsymbol{\theta}, \boldsymbol{\psi}, \boldsymbol{\xi}, \mathbf{x}\right) \\
& =f_{\mathbf{E}^{L_{1} \mid \Psi}}\left(\mathbf{y}^{L_{1}}-\mathbf{y}_{M}^{L_{1}}(\mathbf{x}, \boldsymbol{\theta})-\mathbf{b}^{L_{1}} \mid \boldsymbol{\psi}\right) \cdot f_{\mathbf{B}_{M}^{L_{1} \mid \boldsymbol{\Xi}}}\left(\mathbf{b}^{L_{1}} \mid \boldsymbol{\xi}, \mathbf{x}\right) .
\end{aligned}
$$

Again, with the simplifying assumptions (6) and (7), the conditional distribution of the bias becomes a multivariate normal distribution with mean

$$
\mathrm{E}\left[\mathbf{B}_{M}^{L_{1}} \mid \mathbf{Y}_{M}^{L_{1}}, \boldsymbol{\Theta}, \boldsymbol{\Psi}, \boldsymbol{\Xi}\right]=\boldsymbol{\Sigma}_{\mathbf{B}_{M}^{L_{1}}}\left(\boldsymbol{\Sigma}_{\mathbf{E}^{L_{1}}}+\boldsymbol{\Sigma}_{\mathbf{B}_{M}^{L_{1}}}\right)^{-1} \cdot\left(\mathbf{y}^{L_{1}}-\mathbf{y}_{M}^{L_{1}}(\mathbf{x}, \boldsymbol{\theta})\right)
$$

and covariance matrix

$$
\operatorname{Var}\left[\mathbf{B}_{M}^{L_{1}} \mid \mathbf{Y}_{M}^{L_{1}}, \boldsymbol{\Theta}, \boldsymbol{\Psi}, \boldsymbol{\Xi}\right]=\boldsymbol{\Sigma}_{\mathbf{B}_{M}^{L_{1}}}\left(\boldsymbol{\Sigma}_{\mathbf{E}^{L_{1}}}+\boldsymbol{\Sigma}_{\mathbf{B}_{M}^{L_{1}}}\right)^{-1} \boldsymbol{\Sigma}_{\mathbf{E}^{L_{1}}}
$$

See Reichert and Schuwirth (2012) for the derivations.

\subsubsection{Prediction}

In addition to the inference of parameters, we are also interested in predictions at unknown points in time and/or space where observations are not available. By introducing the prediction layout $L_{2}$ and 
the joint layout $L_{1,2}=L_{1} \cup L_{2}$ and using the likelihood function (5), we get the following expression for observed outputs at the new layout $L_{2}$ given the observations at layout $L_{1}$ and the model parameters:

$$
f_{\mathbf{Y}_{M}^{L_{2}} \mid \mathbf{Y}_{M}^{L_{1}}, \boldsymbol{\Theta}, \mathbf{\Psi}, \boldsymbol{\Xi}}\left(\mathbf{y}^{L_{2}} \mid \mathbf{y}^{L_{1}}, \boldsymbol{\theta}, \boldsymbol{\psi}, \boldsymbol{\xi}, \mathbf{x}\right)=\frac{f_{\mathbf{Y}_{M}^{L_{1,2} \mid \boldsymbol{\Theta}, \boldsymbol{\Psi}, \boldsymbol{\Xi}}}\left(\mathbf{y}^{L_{1,2}} \mid \boldsymbol{\theta}, \boldsymbol{\psi}, \boldsymbol{\xi}, \mathbf{x}\right)}{f_{\mathbf{Y}_{M}^{L_{1} \mid \boldsymbol{\Theta}, \boldsymbol{\Psi}, \boldsymbol{\Xi}}}\left(\mathbf{y}^{L_{1}} \mid \boldsymbol{\theta}, \boldsymbol{\psi}, \boldsymbol{\xi}, \mathbf{x}\right)}
$$

The density of the predictions not conditional on model parameters is obtained by multiplying this expression with the posterior density (11) of the parameters for layout $L_{1}$ and integrating out the parameters:

$$
\begin{aligned}
f_{\mathbf{Y}_{M}^{L_{2}} \mid \mathbf{Y}_{M}^{L_{1}}}\left(\mathbf{y}^{L_{2}} \mid \mathbf{y}^{L_{1}}, \mathbf{x}\right) \\
\quad=\int f_{\mathbf{Y}_{M}^{L_{2}} \mid \mathbf{Y}_{M}^{L_{1}, \boldsymbol{\Theta}, \boldsymbol{\Psi}, \boldsymbol{\Xi}}}\left(\mathbf{y}^{L_{2}} \mid \mathbf{y}^{L_{1}}, \boldsymbol{\theta}, \boldsymbol{\psi}, \boldsymbol{\xi}, \mathbf{x}\right) \cdot f_{\boldsymbol{\Theta}, \boldsymbol{\Psi}, \boldsymbol{\Xi} \mid \mathbf{Y}_{M}^{L_{1}}}\left(\boldsymbol{\theta}, \boldsymbol{\psi}, \boldsymbol{\xi} \mid \mathbf{y}^{L_{1}}, \mathbf{x}\right) \mathrm{d} \boldsymbol{\theta} \mathrm{d} \boldsymbol{\psi} \mathrm{d} \boldsymbol{\xi}
\end{aligned}
$$

Using the likelihood (8) and equation (16) leads to a normal distribution for predicted observations at layout $L_{2}$, given observations at layout $L_{1}$ and model parameters, with mean

$$
\mathrm{E}\left[\mathbf{Y}_{M}^{L_{2}} \mid \mathbf{Y}_{M}^{L_{1}}, \boldsymbol{\Theta}, \boldsymbol{\Psi}, \boldsymbol{\Xi}\right]=\mathbf{y}_{M}^{L_{2}}(\mathbf{x}, \boldsymbol{\theta})+\left(\boldsymbol{\Sigma}_{\mathbf{E}^{L_{1,2}}}+\boldsymbol{\Sigma}_{\mathbf{B}_{M}^{L_{1,2}}}\right)_{L_{2}, L_{1}}\left(\boldsymbol{\Sigma}_{\mathbf{E}^{L_{1}}}+\boldsymbol{\Sigma}_{\mathbf{B}_{M}^{L_{1}}}\right)^{-1}\left(\mathbf{y}^{L_{1}}-\mathbf{y}_{M}^{L_{1}}(\mathbf{x}, \boldsymbol{\theta})\right)
$$

and variance-covariance matrix

$$
\begin{aligned}
& \operatorname{Var}\left[\mathbf{Y}_{M}^{L_{2}} \mid \mathbf{Y}_{M}^{L_{1}}, \boldsymbol{\Theta}, \boldsymbol{\Psi}, \boldsymbol{\Xi}\right] \\
& \quad=\boldsymbol{\Sigma}_{\mathbf{E}^{L_{2}}}+\boldsymbol{\Sigma}_{\mathbf{B}_{M}^{L_{2}}}-\left(\boldsymbol{\Sigma}_{\mathbf{E}^{L_{1,2}}}+\boldsymbol{\Sigma}_{\mathbf{B}_{M}^{L_{1,2}}}\right)_{L_{2}, L_{1}}\left(\boldsymbol{\Sigma}_{\mathbf{E}^{L_{1}}}+\boldsymbol{\Sigma}_{\mathbf{B}_{M}^{L_{1}}}\right)^{-1}\left(\boldsymbol{\Sigma}_{\mathbf{E}^{L_{1,2}}}+\boldsymbol{\Sigma}_{\mathbf{B}_{M}^{L_{1,2}}}\right)_{L_{2}, L_{1}}^{\mathrm{T}}
\end{aligned}
$$

Again, we are interested in the contribution of the bias. Its conditional distribution becomes a multivariate normal distribution with mean

$$
\mathrm{E}\left[\mathbf{B}_{M}^{L_{2}} \mid \mathbf{Y}_{M}^{L_{1}}, \boldsymbol{\Theta}, \boldsymbol{\Psi}, \boldsymbol{\Xi}\right]=\left(\boldsymbol{\Sigma}_{\mathbf{B}_{M}^{L_{1,2}}}\right)_{L_{2}, L_{1}}\left(\boldsymbol{\Sigma}_{\mathbf{E}^{L_{1}}}+\boldsymbol{\Sigma}_{\mathbf{B}_{M}^{L_{1}}}\right)^{-1}\left(\mathbf{y}^{L_{1}}-\mathbf{y}_{M}^{L_{1}}(\mathbf{x}, \boldsymbol{\theta})\right)
$$

and covariance matrix

$$
\operatorname{Var}\left[\mathbf{B}_{M}^{L_{2}} \mid \mathbf{Y}_{M}^{L_{1}}, \boldsymbol{\Theta}, \boldsymbol{\Psi}, \boldsymbol{\Xi}\right]=\boldsymbol{\Sigma}_{\mathbf{B}_{M}^{L_{2}}}-\left(\boldsymbol{\Sigma}_{\mathbf{B}_{M}^{L_{1,2}}}\right)_{L_{2}, L_{1}}\left(\boldsymbol{\Sigma}_{\mathbf{E}^{L_{1}}}+\boldsymbol{\Sigma}_{\mathbf{B}_{M}^{L_{1}}}\right)^{-1}\left(\boldsymbol{\Sigma}_{\mathbf{B}_{M}^{L_{1,2}}}\right)_{L_{2}, L_{1}}^{\mathrm{T}}
$$

The final predictive density for $\mathbf{Y}_{M}^{L_{2}} \mid \mathbf{Y}_{M}^{L_{1}}$ only conditional on given observations can be obtained by substituting the normal distributions with the mean and variance-covariance matrix from equations 
(18) and (19) into equation (17):

$$
\begin{aligned}
& f_{\mathbf{Y}_{M}^{L_{2}} \mid \mathbf{Y}_{M}^{L_{1}}}\left(\mathbf{y}^{L_{2}} \mid \mathbf{y}^{L_{1}}, \mathbf{x}\right)=\frac{1}{\sqrt{2 \pi}^{n_{L_{2}}}} \int \frac{f_{\boldsymbol{\Theta}, \boldsymbol{\Psi}, \boldsymbol{\Xi} \mid \mathbf{Y}_{M}^{L_{1}}}\left(\boldsymbol{\theta}, \boldsymbol{\psi}, \boldsymbol{\xi} \mid \mathbf{y}^{L_{1}}, \mathbf{x}\right)}{\sqrt{\operatorname{det}\left(\operatorname{Var}\left[\mathbf{Y}_{M}^{L_{2}} \mid \mathbf{Y}_{M}^{L_{1}}, \boldsymbol{\Theta}, \boldsymbol{\Psi}, \boldsymbol{\Xi}\right]\right)}} \\
& \cdot \exp \left(-\frac{1}{2}\left(\mathbf{y}^{L_{2}}-\mathrm{E}\left[\mathbf{Y}_{M}^{L_{2}} \mid \mathbf{Y}_{M}^{L_{1}}, \boldsymbol{\Theta}, \mathbf{\Psi}, \boldsymbol{\Xi}\right]\right)^{\mathrm{T}} \cdot \operatorname{Var}\left[\mathbf{Y}_{M}^{L_{2}} \mid \mathbf{Y}_{M}^{L_{1}}, \boldsymbol{\Theta}, \mathbf{\Psi}, \boldsymbol{\Xi}\right]^{-1}\right. \\
& \left.\cdot\left(\mathbf{y}^{L_{2}}-\mathrm{E}\left[\mathbf{Y}_{M}^{L_{2}} \mid \mathbf{Y}_{M}^{L_{1}}, \boldsymbol{\Theta}, \boldsymbol{\Psi}, \boldsymbol{\Xi}\right]\right)\right) \mathrm{d} \boldsymbol{\theta} \mathrm{d} \boldsymbol{\psi} \mathrm{d} \boldsymbol{\xi} .
\end{aligned}
$$

We may not only be interested in predicting new observations, $\mathbf{Y}_{M}^{L_{2}}$, but predicting our knowledge of

the true values represented by $\mathbf{y}_{M}^{L_{2}}+\mathbf{B}_{M}^{L_{2}}$. This leads to the same expressions as equations 18 - 222 with setting all terms $\boldsymbol{\Sigma}_{\mathbf{E}^{L_{i}}}$ to zero.

\subsubsection{Link to Multi-Objective Calibration}

The statistical bias description technique outlined in the preceding section was then linked to multiobjective model calibration (Yapo et al. 1998; Gupta et al. 1998, Madsen 2000 Madsen et al. 2002 Gupta et al. 2003; Boyle et al. 2003, Savić et al. 2011) by Reichert and Schuwirth (2012) by realizing that the prior of the bias can be used to influence how the overall bias will be "distributed" among different model output variables.

\subsection{Transformation}

To account for heteroscedasticity in model output, we can apply the technique described in section 2.1 to Box-Cox transformed data and model results (Box and Cox 1964; Box and Cox 1982). Forward and backward Box-Cox transformations can be formulated as

$$
\begin{aligned}
& g(y)= \begin{cases}\frac{\left(y+\lambda_{2}\right)^{\lambda_{1}}-1}{\lambda_{1}} & \lambda_{1} \neq 0 \\
\ln \left(y+\lambda_{2}\right) & \lambda_{1}=0\end{cases} \\
& g^{-1}(z)= \begin{cases}\left(\lambda_{1} z+1\right)^{1 / \lambda_{1}}-\lambda_{2} & \lambda_{1} \neq 0 \\
\exp (z)-\lambda_{2} & \lambda_{1}=0\end{cases} \\
& \frac{\mathrm{d} g}{\mathrm{~d} y}=\left(y+\lambda_{2}\right)^{\lambda_{1}-1}
\end{aligned}
$$

In this equation, $y$ represents a component of the vector $\mathbf{y}^{L}$ and the Box-Cox transformation parameters $\lambda_{1}$ and $\lambda_{2}$ can be chosen to best reduce the heteroscedasticity of $\mathbf{y}^{L}$. This leads to the 
following modification of the error model (4)

$$
\mathbf{g}\left(\mathbf{Y}_{M}^{L}(\mathbf{x}, \boldsymbol{\theta}, \boldsymbol{\psi}, \boldsymbol{\xi})\right)=\mathbf{g}\left(\mathbf{y}_{M}^{L}(\mathbf{x}, \boldsymbol{\theta})\right)+\tilde{\mathbf{B}}_{M}^{L}(\mathbf{x}, \boldsymbol{\xi})+\tilde{\mathbf{E}}^{L}(\boldsymbol{\psi})
$$

where $\mathbf{g}$ indicates application of the transformation $(23)$ to all components of its argument. $\tilde{\mathbf{B}}$ and $\tilde{\mathbf{E}}$ represent bias and measurement error on a transformed scale at which they are additive to the transformed model results. Through backward transformation of the results we get an equation for non-transformed model results as follows

$$
\mathbf{Y}_{M}^{L}(\mathbf{x}, \boldsymbol{\theta}, \boldsymbol{\psi}, \boldsymbol{\xi})=\mathbf{g}^{-1}\left(\mathbf{g}\left(\mathbf{y}_{M}^{L}(\mathbf{x}, \boldsymbol{\theta})\right)+\tilde{\mathbf{B}}_{M}^{L}(\mathbf{x}, \boldsymbol{\xi})+\tilde{\mathbf{E}}^{L}(\boldsymbol{\psi})\right)
$$

In this equation $\mathbf{g}^{-1}$ indicates application of the inverse Box-Cox transformation to all components of its argument. All equations described in section 2.1.1 2.1.3 are still valid for the transformed case by exchanging the results of the deterministic model and the observations for their Box-Cox transformed values. In addition, probability densities must be multiplied with the derivative of the Box-Cox transformation to account for the back-transformation to original units. For the likelihood function (8) this leads to:

$$
\begin{aligned}
& f_{\mathbf{Y}_{M}^{L} \mid \boldsymbol{\Theta}, \boldsymbol{\Psi}, \boldsymbol{\Xi}}\left(\mathbf{y}^{L} \mid \boldsymbol{\theta}, \boldsymbol{\psi}, \boldsymbol{\xi}, \mathbf{x}\right)=\frac{1}{\sqrt{2 \pi}^{n_{L}}} \frac{1}{\sqrt{\operatorname{det}\left(\boldsymbol{\Sigma}_{\tilde{\mathbf{E}}^{L}}+\boldsymbol{\Sigma}_{\tilde{\mathbf{B}}_{M}^{L}}\right)}} \\
& \cdot \exp \left(-\frac{1}{2}\left[\mathbf{g}\left(\mathbf{y}^{L}\right)-\mathbf{g}\left(\mathbf{y}_{M}^{L}(\mathbf{x}, \boldsymbol{\theta})\right)\right]^{\mathrm{T}}\left(\boldsymbol{\Sigma}_{\tilde{\mathbf{E}}^{L}}+\boldsymbol{\Sigma}_{\tilde{\mathbf{B}}_{M}^{L}}\right)^{-1}\left[\mathbf{g}\left(\mathbf{y}^{L}\right)-\mathbf{g}\left(\mathbf{y}_{M}^{L}(\mathbf{x}, \boldsymbol{\theta})\right)\right]\right) \cdot \prod_{i=1}^{n_{L}}\left|\frac{\mathrm{d} g}{\mathrm{~d} y}\left(y_{i}^{L}\right)\right| .
\end{aligned}
$$

As the derivatives of the Box-Cox transformation are evaluated at the observations, the last term is only a constant factor that is not relevant when typical numerical schemes for Bayesian inference are applied that do not rely on the normalization of the posterior.

\section{$2.3 \quad$ Numerical Implementation}

Due to the analytical integration over the bias, the calibration technique described in section 2.1 is relatively efficient. In comparison to a calibration technique that ignores bias, it only requires to include some more parameters (of the stochastic process characterizing the bias) to be included into the calibration process and to replace the likelihood function. The bias can then be inferred separately without the need for additional simulations. The numerical implementation of this technique as described by Reichert and Schuwirth (2012) requires an optimization of the posterior followed by a Markov chain simulation of the posterior. This is the most straightforward way of getting a sample from the posterior without further approximation. However, as such a Markov chain sample typically requires tens of thousands of simulation runs, this can still be very difficult to do for computationally 
demanding models. To provide a solution to this problem, it is the goal of this paper to derive an approximate version of this technique that is computationally less demanding. The underlying idea is to derive a normal approximation at the maximum of the posterior and propagate it through linearized error propagation to the results. The required techniques and the guiding equations will be discussed in this section.

\subsubsection{Inference}

To infer the parameters, $\boldsymbol{\Gamma}=(\boldsymbol{\Theta}, \mathbf{\Psi}, \boldsymbol{\Xi})$, we first maximize the posterior given by equation $(12$ for the observation layout $L_{1}$. This leads to a numerical approximation to its mode:

$$
\boldsymbol{\gamma}^{0}\left(\mathbf{y}^{L_{1}}\right) \approx \operatorname{argmax}_{\boldsymbol{\gamma}}\left(\log \left(f_{\mathbf{Y}_{M}^{L_{1}} \mid \boldsymbol{\Gamma}}\left(\mathbf{y}^{L_{1}}, \boldsymbol{\gamma}, \mathbf{x}\right) f_{\mathrm{pri}}(\boldsymbol{\gamma})\right)\right)
$$

This is a prerequisite for any of the numerical inference techniques (for the Markov chain simulation it increases the numerical efficiency by avoiding a potentially slow convergence during a burn-in phase; for the approximate technique suggested in this paper it defines the point in the environment of which a local approximation is to be found). In order to account for the generally poor knowledge of the position of the maximum and the potentially complicated shape of the posterior (Gupta et al. 2003), a global optimization algorithm, such as the Shuffled Complex Evolution (SCE) Algorithm (Duan et al. 1993 ) or the Particle Swarm Optimization (PSO) algorithm (Kennedy and Eberhart 1995 . Trelea 2003 should be used to get a reasonable approximation of $\gamma^{0}$.

In environmental modeling, the shape of the posterior may be complicated (Gupta et al. 2003). This makes it challenging to get a good approximation by a Normal distribution, $\mathrm{N}\left(\mathrm{E}_{\boldsymbol{\Gamma}}, \operatorname{Var}_{\boldsymbol{\Gamma}}\right)$. For this reason, we suggest to apply different approximation techniques and analyze their behavior for the specific application at hand.

\section{Finite Difference Approximation}

The most straightforward way of getting a Normal approximation at the maximum of the posterior is to derive the variance-covariance matrix from the curvature of the posterior at its maximum by

$$
\operatorname{Var}_{\boldsymbol{\Gamma}} \approx-\left(\left.\frac{\partial^{2} \log \left(f_{\mathrm{post}}\left(\boldsymbol{\gamma}, \mathbf{y}^{L_{1}}, \mathbf{x}\right)\right)}{\partial \boldsymbol{\gamma}^{\mathrm{T}} \partial \boldsymbol{\gamma}}\right|_{\boldsymbol{\gamma}=\boldsymbol{\gamma}^{0}}\right)^{-1}=-\left(\left.\frac{\partial^{2} \log \left(f_{\mathbf{Y}_{M}^{L_{1}} \mid \boldsymbol{\Gamma}}\left(\mathbf{y}^{L_{1}}, \boldsymbol{\gamma}, \mathbf{x}\right) f_{\mathrm{pri}}(\boldsymbol{\gamma})\right)}{\partial \boldsymbol{\gamma}^{\mathrm{T}} \partial \boldsymbol{\gamma}}\right|_{\boldsymbol{\gamma}=\boldsymbol{\gamma}^{0}}\right)^{-1}
$$

(Gelman et al. 1995). The approximate distribution is then given by the Normal distribution with this variance-covariance matrix centered at the (approximate) maximum: $\mathrm{N}\left(\mathrm{E}_{\boldsymbol{\Gamma}}=\boldsymbol{\gamma}^{0}, \operatorname{Var}_{\boldsymbol{\Gamma}}\right)$. 
The Hessian in equation $(28)$ can be calculated by the finite difference approximation

$$
\begin{aligned}
& \log \left(f_{\mathbf{Y}_{M}^{L_{1} \mid \boldsymbol{\Gamma}}}\left(\mathbf{y}^{L_{1}}, \boldsymbol{\gamma}^{0}+\delta_{i} \mathbf{e}_{i}+\delta_{j} \mathbf{e}_{j}, \mathbf{x}\right) f_{\mathrm{pri}}\left(\gamma^{0}+\delta_{i} \mathbf{e}_{i}+\delta_{j} \mathbf{e}_{j}\right)\right) \\
& -\log \left(f_{\mathbf{Y}_{M}^{L_{1}} \mid \boldsymbol{\Gamma}}\left(\mathbf{y}^{L_{1}}, \boldsymbol{\gamma}^{0}+\delta_{i} \mathbf{e}_{i}-\delta_{j} \mathbf{e}_{j}, \mathbf{x}\right) f_{\mathrm{pri}}\left(\boldsymbol{\gamma}^{0}+\delta_{i} \mathbf{e}_{i}-\delta_{j} \mathbf{e}_{j}\right)\right) \\
& -\log \left(f_{\mathbf{Y}_{M}^{L_{1}} \mid \boldsymbol{\Gamma}}\left(\mathbf{y}^{L_{1}}, \boldsymbol{\gamma}^{0}-\delta_{i} \mathbf{e}_{i}+\delta_{j} \mathbf{e}_{j}, \mathbf{x}\right) f_{\mathrm{pri}}\left(\boldsymbol{\gamma}^{0}-\delta_{i} \mathbf{e}_{i}+\delta_{j} \mathbf{e}_{j}\right)\right) \\
& \left.\frac{\partial^{2} \log \left(f_{\text {post }}\left(\boldsymbol{\gamma}, \mathbf{y}^{L_{1}}, \mathbf{x}\right)\right)}{\partial \gamma_{i} \partial \gamma_{j}}\right|_{\boldsymbol{\gamma}=\boldsymbol{\gamma}^{0}} \approx \frac{+\log \left(f_{\mathbf{Y}_{M}^{L_{1}} \mid \boldsymbol{\Gamma}}\left(\mathbf{y}^{L_{1}}, \boldsymbol{\gamma}^{0}-\delta_{i} \mathbf{e}_{i}-\delta_{j} \mathbf{e}_{j}, \mathbf{x}\right) f_{\text {pri }}\left(\boldsymbol{\gamma}^{0}-\delta_{i} \mathbf{e}_{i}-\delta_{j} \mathbf{e}_{j}\right)\right)}{4 \delta_{i} \delta_{j}}
\end{aligned}
$$

where $\mathbf{e}_{i}$ is the unit vector in $i$-direction, $\mathbf{e}_{i}=(0, \ldots, 0, \underbrace{1}_{i}, 0, \ldots, 0)^{\mathrm{T}}$, and $\delta_{i}$ is a "small" increment adapted to the scale of $\gamma_{i}($ Gelman et al. 1995).

Note that the calculation of the derivatives in the equations $(28)$ or $(29)$ does not depend on an additive constant to the log of the posterior density and thus does not require normalization.

If there are $n$ parameters to be estimated, this method requires $2 n^{2}$ simulations to be done in addition to the base simulation at the maximum, $\gamma^{0}$, that was already done by the maximization algorithm. It further requires the numerical approximation to the maximum to be accurate enough that the $2 n^{2}$ simulations all lead to smaller posterior values than the base simulation. Given the potentially difficult shape of the posterior (Gupta et al. 2003) this may already be challenging. In addition, such a local approximation may underestimate the variances of the parameters as a local maximum may have a substantially higher curvature than would have an approximate distribution that smoothes the posterior density regionally. Nevertheless, this technique is one of the candidate techniques to be tested for the derivation of the coefficients of a local Normal approximation to the posterior.

\section{Density Fitting}

To avoid the potential problems of a local approximation mentioned above, it would be an alternative to draw a sample of parameter values in the neighborhood of the maximum, $\gamma^{0}$, and to fit the log of a multivariate Normal density to the corresponding (non-normalized) log posterior values of the model. We performed this fit by minimizing the sum of squared deviations between the log posterior values calculated for the sample and the log posterior values approximated by the multivariate Normal density at the same points. We used the Nelder-Mead optimization algorithm to execute this task. If the parameter vector of the model, $\boldsymbol{\Gamma}$, is of length $n$, this requires us to estimate $n(n+3) / 2+1$ parameters of the approximate posterior ( $n$ means, $n$ standard deviations, $n(n-1) / 2$ correlation coefficients, and 1 normalization coefficient) from the log posterior values of the sample. If $n$ is not small, estimating such a large number of distribution parameters may be a challenging problem. This problem becomes even more challenging having the constraints in mind: standard deviations must be positive and the correlation coefficients must be between minus unity and unity and lead to a positive 
definite correlation matrix. On the other hand, as this technique avoids the problems of the local approximation mentioned above, it may be worth testing. To decrease the number of distribution parameters to be estimated, an alternative might be to keep the mean fixed at the maximum of the sample and recalculate the normalization coefficient in each fitting iteration. This decreases the number of distribution parameters to be estimated to $n(n+1) / 2$.

\section{Importance Sampling}

Given the potential difficulties of a local normal approximation and those of a regional fit of a Normal density mentioned in the previous paragraphs, an alternative concept of getting a Normal approximation may be needed. Importance sampling may be such an alternative. Importance sampling (Geweke 1989; Gelman et al. 1995) is a technique to calculate weights to a sample of a sampling distribution that make it a weighted sample of another distribution, in our case the posterior. In principle, the sampling distribution is arbitrary (except that the density should not be zero within the range of the posterior), but the technique is only efficient if the sampling distribution is a reasonable approximation to the posterior. The weighted sample of the posterior can then be the basis for calculating the posterior mean, $\mathrm{E}_{\boldsymbol{\Gamma}}$, and variance-covariance matrix, $\operatorname{Var}_{\boldsymbol{\Gamma}}$, that can then be used as the parameters of a Normal distribution that approximates the posterior. This leads again to a regional approximation, but the advantage is that the derivation of the mean and variance-covariance matrix does not depend on the assumption of normality. This makes the technique more robust than the previously discussed techniques. Normality is still assumed in the next step of using a Normal distribution with this mean and this variance-covariance matrix as a basis for the subsequent step of (linearized) error propagation.

Technically, a sample, $\left\{\gamma^{(i)}\right\}_{i=1}^{N}$, with sample size $N$, is drawn from a distribution with density $f_{\text {samp }}$ centered at $\boldsymbol{\gamma}^{0}$ and then weights are calculated according to

$$
w_{\text {post }, i}=\frac{\frac{f_{\mathbf{Y}_{M}^{L_{1}} \mid \boldsymbol{\Gamma}}\left(\mathbf{y}^{L_{1}}, \boldsymbol{\gamma}^{(i)}, \mathbf{x}\right) f_{\mathrm{pri}}\left(\boldsymbol{\gamma}^{(i)}\right)}{f_{\text {samp }}\left(\boldsymbol{\gamma}^{(i)}\right)}}{\sum_{j=1}^{N} \frac{f_{\mathbf{Y}_{M}^{L_{1}} \mid \boldsymbol{\Gamma}}\left(\mathbf{y}^{L_{1}}, \boldsymbol{\gamma}^{(j)}, \mathbf{x}\right) f_{\mathrm{pri}}\left(\boldsymbol{\gamma}^{(j)}\right)}{f_{\mathrm{samp}}\left(\boldsymbol{\gamma}^{(j)}\right)}} .
$$

Mean and variance-covariance matrix are then calculated using the sample $\left\{\gamma^{(i)}\right\}_{i=1}^{N}$ with weights $\left\{w_{\text {post }, i}\right\}_{i=1}^{N}$ and the Normal distribution with this mean and this variance-covariance matrix is used to approximate the posterior. The procedure may be iterated by sampling from this Normal distribution and derive new weights to get new estimates of mean and variance-covariance matrix that are based on this sampling distribution that should be a better approximation to the posterior (Reichert et al. 2002).

We suggest two strategies of finding a reasonable sampling distribution. The first consists of guessing reasonable standard deviations of the parameters (e.g. a constant fraction of the parameter 
values) and choosing a Normal sampling distribution centered at $\gamma^{0}$ with these standard deviations and without correlations as a first sampling distribution. The sampling distribution could then be replaced iteratively by the Normal distribution with mean and variance-covariance matrix derived from the weighted sample. If it seems too difficult to guess standard deviations for the first sampling distribution, a uniform distribution in a ball around the maximum could be chosen as a first sampling distribution (see Chen (2011) for how to sample from a uniform distribution in a ball). The radius of the ball should not be too small to avoid severe bias by the property of the density of this distribution to be zero outside the ball (see comment above about importance sampling). The estimates of mean and variance-covariance matrix could be improved subsequently by proceeding as above with a Normal sampling distribution with parameters derived from the previous step. This second procedure can be expected to be less efficient regarding the effective sample size of the first step (based on the uniform sample in the ball), but more robust regarding the potential of identifying a higher posterior density maximum than the one calculated by the optimization algorithm.

\subsubsection{Prediction}

The different approximation techniques described in section 2.3.1 result in the parameters $\mathrm{E}_{\boldsymbol{\Gamma}}$ and $\operatorname{Var}_{\boldsymbol{\Gamma}}$ of a Normal distribution approximating the posterior distribution. These parameters can be used for a propagation of this posterior through the model for an uncertainty analysis of the model for a prediction time. Given a conditional probability density of results given parameters, $f_{\mathbf{Y} \mid \boldsymbol{\Gamma}}$, and a probability density of the parameters, $f_{\Gamma}$, we can calculate the unconditional predictions of the results by marginalization of the joint density as

$$
f_{\mathbf{Y}}(\mathbf{y})=\int f_{\mathbf{Y} \mid \boldsymbol{\Gamma}}(\mathbf{y} \mid \gamma) \cdot f_{\Gamma}(\gamma) \mathrm{d} \boldsymbol{\gamma}
$$

If both distributions, $f_{\mathbf{Y} \mid \boldsymbol{\Gamma}}$, and $f_{\boldsymbol{\Gamma}}$ are Normal and we can neglect the dependence of $\operatorname{det}\left[\operatorname{Var}_{\mathbf{Y} \mid \boldsymbol{\Gamma}}\right]$ on the parameter vector $\boldsymbol{\gamma}=(\boldsymbol{\theta}, \boldsymbol{\psi}, \boldsymbol{\xi})$ in the normalization constant, we get the approximate proportionality

$f_{\mathbf{Y}}(\mathbf{y}) \propto \int \exp \left(-\frac{1}{2}\left[\mathbf{y}-\mathrm{E}_{\mathbf{Y} \mid \boldsymbol{\Gamma}}(\boldsymbol{\gamma})\right]^{\mathrm{T}} \operatorname{Var}_{\mathbf{Y} \mid \boldsymbol{\Gamma}}(\boldsymbol{\gamma})^{-1}\left[\mathbf{y}-\mathrm{E}_{\mathbf{Y} \mid \boldsymbol{\Gamma}}(\boldsymbol{\gamma})\right]-\frac{1}{2}\left(\boldsymbol{\gamma}-\mathrm{E}_{\boldsymbol{\Gamma}}\right)^{\mathrm{T}} \operatorname{Var}_{\boldsymbol{\Gamma}}^{-1}\left(\boldsymbol{\gamma}-\mathrm{E}_{\boldsymbol{\Gamma}}\right)\right) \mathrm{d} \boldsymbol{\gamma}$

If we further approximate this expression by neglecting the dependence of $\operatorname{Var}_{\mathbf{Y} \mid \boldsymbol{\Gamma}}$ on $\gamma$ and approximate the dependence of $\mathrm{E}_{\mathbf{Y} \mid \boldsymbol{\Gamma}}$ on $\gamma$ by the constant and linear terms of the Taylor series, we obtain 
the approximation

$$
\begin{aligned}
f_{\mathbf{Y}}(\mathbf{y}) \propto \int \exp (- & \frac{1}{2}\left[\mathbf{y}-\mathrm{E}_{\mathbf{Y} \mid \boldsymbol{\Gamma}}\left(\mathrm{E}_{\boldsymbol{\Gamma}}\right)-\frac{\partial \mathrm{E}_{\mathbf{Y} \mid \boldsymbol{\Gamma}}}{\partial \boldsymbol{\gamma}^{\mathrm{T}}}\left(\boldsymbol{\gamma}-\mathrm{E}_{\boldsymbol{\Gamma}}\right)\right]^{\mathrm{T}} \operatorname{Var}_{\mathbf{Y} \mid \boldsymbol{\Gamma}}\left(\mathrm{E}_{\boldsymbol{\Gamma}}\right)^{-1} \\
\cdot & {\left.\left[\mathbf{y}-\mathrm{E}_{\mathbf{Y} \mid \boldsymbol{\Gamma}}\left(\mathrm{E}_{\boldsymbol{\Gamma}}\right)-\frac{\partial \mathrm{E}_{\mathbf{Y} \mid \boldsymbol{\Gamma}}}{\partial \boldsymbol{\gamma}^{\mathrm{T}}}\left(\boldsymbol{\gamma}-\mathrm{E}_{\boldsymbol{\Gamma}}\right)\right]-\frac{1}{2}\left(\boldsymbol{\gamma}-\mathrm{E}_{\boldsymbol{\Gamma}}\right)^{\mathrm{T}} \operatorname{Var}_{\boldsymbol{\Gamma}}^{-1}\left(\boldsymbol{\gamma}-\mathrm{E}_{\boldsymbol{\Gamma}}\right)\right) \mathrm{d} \boldsymbol{\gamma} }
\end{aligned}
$$

When completing the square to get a Normal density in $\gamma$, integrating over $\gamma$ and completing the square a second time to get a Normal density in $\mathbf{y}$, we finally get a Normal distribution for $\mathbf{Y}$ with mean $\mathrm{E}_{\mathbf{Y} \mid \boldsymbol{\Gamma}}\left(\mathrm{E}_{\boldsymbol{\Gamma}}\right)$ and variance:

$$
\begin{gathered}
\operatorname{Var}_{\mathbf{Y}}=\left[\operatorname{Var}_{\mathbf{Y} \mid \boldsymbol{\Gamma}}\left(\mathrm{E}_{\boldsymbol{\Gamma}}\right)^{-1}-\operatorname{Var}_{\mathbf{Y} \mid \boldsymbol{\Gamma}}\left(\mathrm{E}_{\boldsymbol{\Gamma}}\right)^{-1} \cdot \frac{\partial \mathrm{E}_{\mathbf{Y} \mid \boldsymbol{\Gamma}}}{\partial \boldsymbol{\gamma}^{\mathrm{T}}}\left(\mathrm{E}_{\boldsymbol{\Gamma}}\right)\right. \\
\left.\cdot\left[\left(\frac{\partial \mathrm{E}_{\mathbf{Y} \mid \boldsymbol{\Gamma}}}{\partial \boldsymbol{\gamma}^{\mathrm{T}}}\left(\mathrm{E}_{\boldsymbol{\Gamma}}\right)\right)^{\mathrm{T}} \cdot \operatorname{Var}_{\mathbf{Y} \mid \boldsymbol{\Gamma}}\left(\mathrm{E}_{\boldsymbol{\Gamma}}\right)^{-1} \cdot \frac{\partial \mathrm{E}_{\mathbf{Y} \mid \boldsymbol{\Gamma}}}{\partial \boldsymbol{\gamma}^{\mathrm{T}}}\left(\mathrm{E}_{\boldsymbol{\Gamma}}\right)+\operatorname{Var}_{\boldsymbol{\Gamma}}^{-1}\right]^{-1} \cdot\left(\frac{\partial \mathrm{E}_{\mathbf{Y} \mid \boldsymbol{\Gamma}}}{\partial \boldsymbol{\gamma}^{\mathrm{T}}}\left(\mathrm{E}_{\boldsymbol{\Gamma}}\right)\right)^{\mathrm{T}} \cdot \operatorname{Var}_{\mathbf{Y} \mid \boldsymbol{\Gamma}}\left(\mathrm{E}_{\boldsymbol{\Gamma}}\right)^{-1}\right]^{-1}
\end{gathered}
$$

This can further be simplified to

$$
\mathrm{E}_{\mathbf{Y}} \approx \mathrm{E}_{\mathbf{Y} \mid \boldsymbol{\Gamma}}\left(\mathrm{E}_{\boldsymbol{\Gamma}}\right)
$$

and

$$
\operatorname{Var}_{\mathbf{Y}} \approx \operatorname{Var}_{\mathbf{Y} \mid \boldsymbol{\Gamma}}\left(\mathrm{E}_{\boldsymbol{\Gamma}}\right)+\frac{\partial \mathrm{E}_{\mathbf{Y} \mid \boldsymbol{\Gamma}}}{\partial \boldsymbol{\gamma}^{\mathrm{T}}}\left(\mathrm{E}_{\boldsymbol{\Gamma}}\right) \cdot \operatorname{Var}_{\boldsymbol{\Gamma}} \cdot\left(\frac{\partial \mathrm{E}_{\mathbf{Y} \mid \boldsymbol{\Gamma}}}{\partial \boldsymbol{\gamma}^{\mathrm{T}}}\left(\mathrm{E}_{\boldsymbol{\Gamma}}\right)\right)^{\mathrm{T}}=\operatorname{Var}_{\mathbf{Y} \mid \boldsymbol{\Gamma}}\left(\mathrm{E}_{\boldsymbol{\Gamma}}\right)+\mathbf{V} \cdot \operatorname{Var}_{\boldsymbol{\Gamma}} \cdot \mathbf{V}^{\mathrm{T}}
$$

with the Jacobian

$$
\mathbf{V}=\frac{\partial \mathrm{E}_{\mathbf{Y} \mid \boldsymbol{\Gamma}}}{\partial \boldsymbol{\gamma}^{\mathrm{T}}}\left(\mathrm{E}_{\boldsymbol{\Gamma}}\right)
$$

This approximation will now be used to get approximate predictions for new observations, $\mathbf{Y}_{M}^{L_{i}}$, and for our knowledge of the true state, $\mathbf{y}_{M}^{L_{i}}+\mathbf{B}_{M}^{L_{i}}$, for both layouts, $L_{1}$ and $L_{2}$, based on the parameters of the conditional Normal distributions discussed in section 2.1.3 and the Normal approximation of the posterior discussed in section 2.3.1. In addition, we are interested in the predictions for the bias, $\mathbf{B}_{M}^{L_{i}}$, and for the posterior of the observation error for layout $L_{1}, \mathbf{E}^{L_{1}}$, to get a probabilistic separation of the residuals into bias and observation error. The Jacobians for these four cases are given by

$$
\mathbf{V}_{\mathbf{Y}_{M}^{L_{1,2}}}=\left.\frac{\partial \mathrm{E}\left[\mathbf{Y}_{M}^{L_{1,2}} \mid \mathbf{Y}_{M}^{L_{1}}, \boldsymbol{\Gamma}\right]}{\partial \boldsymbol{\gamma}^{\mathrm{T}}}\right|_{\boldsymbol{\gamma}=\boldsymbol{\gamma}^{0}}
$$


using the expected value as presented in equation (18),

$$
\mathbf{V}_{\mathbf{y}_{M}^{L_{1,2}}+\mathbf{B}_{M}^{L_{1,2}}}=\left.\frac{\partial \mathrm{E}\left[\mathbf{y}_{M}^{L_{1,2}}+\mathbf{B}_{M}^{L_{1,2}} \mid \mathbf{Y}_{M}^{L_{1}}, \boldsymbol{\Gamma}\right]}{\partial \boldsymbol{\gamma}^{T}}\right|_{\boldsymbol{\gamma}=\boldsymbol{\gamma}^{0}}=\left.\frac{\partial\left(\mathbf{y}_{M}^{L_{1,2}}+\mathrm{E}\left[\mathbf{B}_{M}^{L_{1,2}} \mid \mathbf{Y}_{M}^{L_{1}}, \boldsymbol{\Gamma}\right]\right)}{\partial \boldsymbol{\gamma}^{T}}\right|_{\boldsymbol{\gamma}=\boldsymbol{\gamma}^{0}}
$$

using (14) and (20),

$$
\mathbf{V}_{\mathbf{B}_{M}^{L_{1,2}}}=\left.\frac{\partial \mathrm{E}\left[\mathbf{B}_{M}^{L_{1,2}} \mid \mathbf{Y}_{M}^{L_{1}}, \boldsymbol{\Gamma}\right]}{\partial \boldsymbol{\gamma}^{\mathrm{T}}}\right|_{\boldsymbol{\gamma}=\boldsymbol{\gamma}^{0}}
$$

using again the equations (14) and (20), and finally

$$
\mathbf{V}_{\mathbf{E}_{M}^{L_{1}}}=\left.\frac{\partial \mathrm{E}\left[\mathbf{E}_{M}^{L_{1}} \mid \mathbf{Y}_{M}^{L_{1}}, \boldsymbol{\Gamma}\right]}{\partial \boldsymbol{\gamma}^{\mathrm{T}}}\right|_{\boldsymbol{\gamma}=\boldsymbol{\gamma}^{0}}=-\left.\frac{\partial \mathrm{E}\left[\mathbf{y}_{M}^{L_{1}}+\mathbf{B}_{M}^{L_{1}} \mid \mathbf{Y}_{M}^{L_{1}}, \boldsymbol{\Gamma}\right]}{\partial \boldsymbol{\gamma}^{\mathrm{T}}}\right|_{\boldsymbol{\gamma}=\boldsymbol{\gamma}^{0}}
$$

which results from equation (4). To apply equations (35) and (36) for calculating the approximate predictions for the four cases $\mathbf{Y}_{M}^{L_{i}}, \mathbf{y}_{M}^{L_{i}}+\mathbf{B}_{M}^{L_{i}}, \mathbf{B}_{M}^{L_{i}}$, and $\mathbf{E}^{L_{1}}$, we need the expected values referenced above, the Jacobians given by the equations (38) to (41), and the variances (19), 19) with ignoring the error terms $\boldsymbol{\Sigma}_{\mathbf{E}^{L_{i}}},(15)$, and again (15), respectively.

To evaluate the expressions (38)-(41), we need the derivatives of the different expected values. In all four cases, these expected values can be written in the form

$$
\mathrm{E}\left[\mathbf{Y} \mid \mathbf{Y}_{M}^{L}, \boldsymbol{\Gamma}\right](\gamma)=\mathbf{a}(\gamma)+\mathbf{A}(\gamma) \cdot \mathbf{y}_{M}^{L}(\gamma)
$$

where a represents a vector and $\mathbf{A}$ a matrix chosen in a way that the three equations (14), (18) and (20) match equation 42). The derivates can be calculated according to

$$
\left.\frac{\partial \mathrm{E}\left[\mathbf{Y} \mid \mathbf{Y}_{M}^{L}, \boldsymbol{\Gamma}\right]}{\partial \gamma_{j}}\right|_{\boldsymbol{\gamma}=\boldsymbol{\gamma}^{0}}=\frac{\partial \mathbf{a}}{\partial \gamma_{j}}\left(\gamma^{0}\right)+\frac{\partial \mathbf{A}}{\partial \gamma_{j}}\left(\gamma^{0}\right) \cdot \mathbf{y}_{M}^{L}\left(\gamma^{0}\right)+\mathbf{A}\left(\boldsymbol{\gamma}^{0}\right) \cdot \frac{\partial \mathbf{y}_{M}^{L}}{\partial \gamma_{j}}\left(\gamma^{0}\right)
$$

As the derivatives of $\mathbf{a}$ and of $\mathbf{A}$ are computationally much less demanding (no simulations of the deterministic model required) than that of $\mathbf{y}_{M}^{L}$, this equation enables to save computation time by using the same Jacobian of $\mathbf{y}_{M}^{L}$ for all four cases mentioned above. In addition it allows us to restrict the calculations of the finite difference approximation of this Jacobian to the components $\boldsymbol{\theta}$ of the parameter vector $\gamma=(\boldsymbol{\theta}, \boldsymbol{\psi}, \boldsymbol{\xi})$ as the results of the deterministic model do not depend on the parameters $\boldsymbol{\psi}$ and $\boldsymbol{\xi}$. When using the Box-Cox transformation (see section 2.2, these equations must be applied to the transformed model results. This requires calculating the Jacobian of transformed results from that of not transformed results

$$
\frac{\partial \mathbf{g}\left(\mathbf{y}_{M}^{L}\right)}{\partial \boldsymbol{\gamma}^{\mathbf{T}}}=\frac{\partial \mathbf{y}_{M}^{L}}{\partial \boldsymbol{\gamma}^{\mathbf{T}}} \cdot \operatorname{diag}\left(\frac{\mathrm{d} \mathbf{g}}{\mathrm{d} y}\left(\mathbf{y}_{M}^{L}\left(\boldsymbol{\gamma}^{0}\right)\right)\right)
$$


where again $\mathbf{g}$ and $\mathrm{d} \mathbf{g} / \mathrm{d} y$ return the vector of results of the application of the functions (23) to all components.

As an example of the complete predictive distribution, we give the distribution of our knowledge of the real state of the system:

$$
\begin{aligned}
\mathbf{y}_{M}^{L_{1,2}}+\mathbf{B}_{M}^{L_{1,2}} \mid \mathbf{Y}_{M}^{L_{1}} \sim & \mathrm{N}\left(\mathbf{y}_{M}^{L_{1,2}}\left(\mathrm{E}_{\boldsymbol{\Gamma}}\right)+\mathrm{E}\left[\mathbf{B}_{M}^{L_{1,2}} \mid \mathbf{Y}_{M}^{L_{1}}, \boldsymbol{\Gamma}\right]\left(\mathbf{y}^{L_{1}}, \mathrm{E}_{\boldsymbol{\Gamma}}\right)\right. \\
& \left.\mathbf{V}_{\mathbf{y}_{M}^{L_{1,2}}+\mathbf{B}_{M}^{L_{1,2}}} \cdot \operatorname{Var}_{\boldsymbol{\Gamma}} \cdot\left(\mathbf{V}_{\mathbf{y}_{M}^{L_{1,2}}+\mathbf{B}_{M}^{L_{1,2}}}\right)^{\mathrm{T}}+\operatorname{Var}\left[\mathbf{B}_{M}^{L_{1,2}} \mid \mathbf{Y}_{M}^{L_{1}}, \boldsymbol{\Gamma}\right]\left(\mathbf{y}^{L_{1}}, \mathrm{E}_{\boldsymbol{\Gamma}}\right)\right)
\end{aligned}
$$

where $\mathrm{E}_{\boldsymbol{\Gamma}}$ and $\operatorname{Var}_{\boldsymbol{\Gamma}}$ are the parameters of the Normal distribution approximating the posterior derived according to one of the techniques described in section 2.3.1. The mean of the bias is calculated according to equations $(14)$ and 200 , its variance according to equations 12 and 21$] . \mathbf{V}_{\mathbf{y}_{M}}^{L_{1,2}}+\mathbf{B}_{M}^{L_{1,2}}$ follows from equation (39) using equations 42 to 44 ).

\subsection{Summary of the Approximate Calibration and Prediction Procedure}

The suggested approximate multi-objective calibration technique including uncertainty analysis of model predictions for a deterministic model $\mathbf{y}_{M}^{L}(\mathbf{x}, \boldsymbol{\theta})$, based on the concepts presented in sections 2.1 to 2.3, can be summarized as follows:

1. Specify a prior for the model parameters $\Theta$ based on present knowledge about (possible) parameter values.

2. Specify a potentially informative prior of the parameters $\boldsymbol{\Psi}$ of the covariance matrix of the observation process by using model-independent knowledge (equation (6)). In the presented case, this step consists of specifying priors for the standard deviations of the observation error of all output variables. In case of heteroscedastic data, a Box-Cox transformation (section 2.2 can be performed. The priors should hence represent knowledge about errors for typical concentrations on a transformed scale.

3. Specify a prior for the parameters $\boldsymbol{\Xi}$ of the Gaussian process characterizing prior knowledge of the bias for an observation layout $L_{1}$ (equations (7) and (10)). In the presented case, this step consists of choosing a prior for the smoothing parameters $\beta$ of the correlation structure and for the standard deviations of the bias in different output variables (equation (10)). The prior of the standard deviations should reflect the degree of bias we are willing to accept and the desire to avoid bias (for example with an exponential distribution with which probability density increases with decreasing value of the standard deviation), hence our calibration objectives, and the expectations about the bias contribution. When applying a Box-Cox transformation, the specification, again, must be done at the transformed scale. 
4. Derive the joint posterior distribution of the parameters $\boldsymbol{\Theta}, \boldsymbol{\Psi}$ and $\boldsymbol{\Xi}$ and the model bias $\mathbf{B}$ by an approximate linearized approach described in section (2.3) using observations $y^{L_{1}}$ of the observation layout $L_{1}$ (equations (12), (14) and (15)). This gives the uncertainty estimation of the model results for times where data provides information about the amount of bias in model results.

5. Quantify the posterior knowledge about the "true" system state, $\mathbf{y}_{M}^{L_{2}}+\mathbf{B}_{M}^{L_{2}} \mid \mathbf{Y}_{M}^{L_{1}}$, and of new observations for the prediction layout $L_{2}$, according to equation 45 without and with considering the observation error and with $\mathrm{E}\left[\mathbf{B}_{M}^{L_{2}} \mid \mathbf{Y}_{M}^{L_{1}}, \boldsymbol{\Theta}, \mathbf{\Psi}, \boldsymbol{\Xi}\right]$ and $\operatorname{Var}\left[\mathbf{B}_{M}^{L_{2}} \mid \mathbf{Y}_{M}^{L_{1}}, \boldsymbol{\Theta}, \mathbf{\Psi}, \boldsymbol{\Xi}\right]$ according to 20 and (21) and $f_{\boldsymbol{\Theta}, \boldsymbol{\Psi}, \boldsymbol{\Xi} \mid \mathbf{Y}_{M}^{L_{1}}}\left(\boldsymbol{\theta}, \boldsymbol{\psi}, \boldsymbol{\xi} \mid \mathbf{y}^{L_{1}}, \mathbf{x}\right)$ according to 12 . This gives the uncertainty estimates of the model results at times where data is not available or not used for calibration. If observations are available for layout $L_{2}$, they can be used for a validation of the predictive power of the model. After conducting the inference and prediction on a Box-Cox transformed scale, for comparison of real data and model output results, the results have to be transformed backward.

\section{Didactical Example}

We use the same model of growth of microbes in a continuously operated, stirred tank reactor as Reichert and Schuwirth (2012), as a simple example for the application of the suggested approach.

\subsection{Data}

In this model, the microbes' concentration, $C_{\mathrm{M}}$, and the substrate concentration, $C_{\mathrm{S}}$, are described by the following mass balance equations:

$$
\begin{aligned}
\frac{\mathrm{d} C_{\mathrm{M}}}{\mathrm{d} t} & =\mu \frac{C_{\mathrm{S}}}{K+C_{\mathrm{S}}} C_{\mathrm{M}} \exp \left(c\left(T-T_{0}\right)\right)-b C_{\mathrm{M}}-q C_{\mathrm{M}} \\
\frac{\mathrm{d} C_{\mathrm{S}}}{\mathrm{d} t} & =-\frac{\mu}{Y} \frac{C_{\mathrm{S}}}{K+C_{\mathrm{S}}} C_{\mathrm{M}} \exp \left(c\left(T-T_{0}\right)\right)+q\left(C_{\mathrm{S}, \text { in }}-C_{\mathrm{S}}\right)
\end{aligned}
$$

with the initial conditions

$$
\begin{aligned}
& C_{\mathrm{M}}(0)=C_{\mathrm{M}, \text { ini }} \\
& C_{\mathrm{S}}(0)=C_{\mathrm{S}, \text { ini }}
\end{aligned}
$$

and the temperature variations

$$
T=T_{0}+A \sin (2 \pi(t-0.25 \mathrm{~d}))
$$

In these equations, $C_{\mathrm{M}}$ is the concentration of microbes in the reactor $\left(\mathrm{ML}^{-3}\right), C_{\mathrm{S}}$ is the concentration of substrate in the reactor $\left(\mathrm{ML}^{-3}\right), t$ is time $(\mathrm{T}), \mu$ is the maximum specific growth rate of the microbes 
$\left(\mathrm{T}^{-1}\right), K$ is the concentration of substrate at which the microbes grow with half of their maximum specific growth rate $\left(\mathrm{ML}^{-3}\right), b$ is the specific death rate of the microbes $\left(\mathrm{T}^{-1}\right), q$ is the volumetric flow rate through the reactor per unit of reactor volume $\left(\mathrm{T}^{-1}\right), Y$ is the yield (produced amount of biomass per consumed amount of substrate) $(-), C_{\mathrm{S} \text {,in }}$ is the concentration of substrate in the inflow $\left(\mathrm{ML}^{-3}\right)$ (the concentration of microbes in the inflow is assumed to be zero), $T$ is temperature, $T_{0}$ is a reference temperature assumed to be 20 degrees Celsius, $A$ is the amplitude of temperature variations, $c$ is a temperature dependence coefficient assumed to be 0.046 per degree Celsius, $C_{\mathrm{M} \text {,ini }}$ is the initial concentration of microbes in the reactor $\left(\mathrm{ML}^{-3}\right)$, and $C_{\mathrm{S} \text {,ini }}$ is the initial concentration of substrate in the reactor $\left(\mathrm{ML}^{-3}\right)$.

We produced synthetic observations with this model for a simulation period of 4 days, with a temperature amplitude of $4^{\circ} \mathrm{C}$ and the following parameter values: $\mu=4, K=10, b=1, q=1, Y=$ 0.6, $C_{\mathrm{S}, \text { in }}=100, C_{\mathrm{M}, \text { ini }}=10, C_{\mathrm{S} \text {,ini }}=40$, and $\sigma_{\mathbf{E}_{C_{\mathrm{M}}}}=0.5$ and $\sigma_{\mathbf{E}_{C_{\mathrm{M}}}}=0.5$ (in model output units) as standard deviations of normally distributed observation errors in both model output variables. The generated data does not contain heteroscedasticity, so no Box-Cox transformation was needed.

\subsection{Model Description}

From the first two days out of these data, we will try to estimate the parameters of a model that describes the same system but is biased because of omitting the temperature dependence of substrate and microbial growth described in equations (46). This represents a typical problem in environmental modeling resulting from choosing a wrong (i.e. simplified) model structure, for example by choosing a wrong set of model equations. With this example we can discuss parameter estimates and model predictions for the full period of four days under the presence of model bias.

Here, the observation layout as introduced in 2 specifies observations of the two variables, $C_{\mathrm{M}}$ and $C_{\mathrm{S}}$, at the time points $\left\{t_{1}, t_{2}, \ldots, t_{n}\right\}$ and given inputs $A$ and parameter $c$. The deterministic model function resulting from the solution of the system of differential equations (46) can be written as

$$
\mathbf{y}_{M}^{L}(\boldsymbol{\theta})=\left(C_{\mathrm{M}}\left(t_{1}, \boldsymbol{\theta}\right), \ldots, C_{\mathrm{M}}\left(t_{n}, \boldsymbol{\theta}\right), C_{\mathrm{S}}\left(t_{1}, \boldsymbol{\theta}\right), \ldots, C_{\mathrm{S}}\left(t_{n}, \boldsymbol{\theta}\right)\right)^{\mathrm{T}}
$$

The vector $\boldsymbol{\theta}=\left(\mu, K, b, q, Y, C_{\mathrm{S}, \text { in }}, C_{\mathrm{M} \text {,ini }}, C_{\mathrm{S} \text {,ini }}\right)^{\mathrm{T}}$ gives the parameter vector of that model. The parameters $\boldsymbol{\psi}=\left(\sigma_{\mathbf{E}_{C_{\mathrm{M}}}}, \sigma_{\mathbf{E}_{C_{\mathrm{S}}}}\right)^{\mathrm{T}}$ consist of the standard deviations $\sigma_{\mathbf{E}_{C_{\mathrm{M}}}}$ and $\sigma_{\mathbf{E}_{C_{\mathrm{S}}}}$ of independent normal distributions characterizing the observation error of $C_{\mathrm{M}}$ and $C_{\mathrm{S}}$ at all points in time. Finally,

the vector $\boldsymbol{\xi}=\left(\sigma_{\mathbf{B}_{C_{\mathrm{M}}}}, \sigma_{\mathbf{B}_{C_{\mathrm{S}}}}, \tau\right)^{\mathrm{T}}$ combines the standard deviations $\sigma_{\mathbf{B}_{C_{\mathrm{M}}}}$ and $\sigma_{\mathbf{B}_{C_{\mathrm{S}}}}$ for the bias in each of the model variables $C_{\mathrm{M}}$ and $C_{\mathrm{S}}$ and the correlation time $\tau$ instead of the parameter $\beta$ (according to equation (7)). The correlation times are kept equal for the two variables in this example but potentially could be different. 


\subsection{Prior Distribution}

As the microbial growth and substrate consumption are coupled by the yield, $Y$ (see the first terms on the right hand sides of the equations (46)) and measured time courses of $C_{\mathrm{M}}$ and $C_{\mathrm{S}}$ might not perfectly match this constraint, the model cannot fit both components equally well. Hence, the quality of fit of each component will depend on the choice of the prior of the bias. Through the choice of the prior of the bias, we can influence how the bias will be "distributed" among the two model variables while an increasing probability density for smaller values of the priors of the standard deviations of the bias should represent the desire to have as little bias as possible. For the priors of $\sigma_{\mathbf{B}_{C_{\mathrm{M}}}}$ and $\sigma_{\mathbf{B}_{C_{\mathrm{S}}}}$, we therefore chose normal distributions with mean zero and a standard deviation of 0.5. They were truncated at zero to avoid negative values. For the parameters $\boldsymbol{\theta}$, we used independent lognormal priors with the means at the correct values given above and standard deviations of $50 \%$. For the parameters $\psi$ and the correlation time $\tau$, lognormal priors of the means at the correct values in case of the observation error parameters (given above) and at 0.3 for $\tau$, which does not have a true value, and standard deviations of $10 \%$ were used. We calibrated the logarithm of all parameters instead of the parameters themselves, as this appeared to lead to a better approximation of the posterior by a Normal distribution. As model parameters are typically positive, this is usually possible. Hence, the posterior marginals of the parameters result in lognormal distributions.

Additionally to this base scenario, we repeated the application of the method for calibration and uncertainty analysis as described in 2 for a second scenario choosing wrong priors, which frequently occurs in the context of environmental modeling. We simulated the choice of wrong priors for the model parameters $\boldsymbol{\theta}$ by setting the mean 0.5 times larger than the correct parameter value. For the results we refer to the supplementary data.

\subsection{Results and Discussion}

In the following we will show the results of the approximate uncertainty analysis as described in 2 for the calibration and the prediction time for the basic prior scenario. Here, we focus on a discussion of the different posterior approximation techniques described in section 2.3 For a discussion of different prior choices for the standard deviations of the bias in $C_{\mathrm{M}}$ and $C_{\mathrm{S}}$ and the effect of ignorance of bias, we refer to Reichert and Schuwirth (2012). The parameter inference procedure described in section 2.3 was performed for this case study based on maximization of the posterior followed by all three approximation techniques described in section 2.3.1. A comparison of the computational burden of each of those techniques compared to the full MCMC approach is given in Table 1 . Depending on the sample size and, for the finite difference and the importance sampling approximation, depending on the number of iterations, it shows a 6-70 times smaller computational demand for the approximation techniques compared to the full MCMC approach. To get a local estimate of the Hessian at the max- 
imum of the posterior, the finite difference technique required us to perform $338\left(=2 \cdot 13^{2}\right)$ simulations in addition to the base simulation at the maximum available from the optimization procedure. Due to the approximate estimation of the position of the maximum, several attempts had to be made to get smaller values of the posterior at these 338 locations than at the numerical "maximum" found so far (by replacing the numerical "maximum" by the position with the higher value and restarting the finite difference approach). This is an indication of a complex posterior shape and/or of a poor performance of the optimization algorithm. Also, the choice of the increment for evaluation of the Hessian is critical, for more information we refer to the supplementary data. The density fitting approach was based on a random sample of 1000 parameter vectors from a uniform distribution in a ball around the numerical approximation to the maximum. Fitting a multivariate Normal distribution (with 105 parameters) to these posterior values failed, as the standard deviations of observation error and/or bias tended to diverge. Finally, importance sampling was also done, based on a sample of size 1000, first from a uniform distribution in a ball around the numerical maximum, then improved by iterative sampling from Normal distributions based on mean and variance-covariance matrix from the previous iteration step until the effective sample size did no longer increase.

Table 1: Number of simulation runs needed for the different approximation techniques and the full Markov chain Monte Carlo method as presented in Reichert and Schuwirth (2012) for the didactical example. All numbers refer to the simulation runs actually performed for the results shown herein. For further improvement some methods could be conducted with larger sample sizes or several iterations of the same technique.

\begin{tabular}{|c|c|c|c|c|c|}
\hline Application Case & Optimization & $\begin{array}{c}\text { Finite } \\
\text { Difference }\end{array}$ & $\begin{array}{l}\text { Density } \\
\text { Fitting }\end{array}$ & $\begin{array}{c}\text { Importance } \\
\text { Sampling }\end{array}$ & $\mathrm{MCMC}$ \\
\hline Didactical Example & $5 \cdot 500[1]$ & $20 \cdot 338^{[2]}$ & 1000 & $11 \cdot 1000[3]$ & $50000(+4 \cdot 5000)^{[4}$ \\
\hline \multicolumn{6}{|c|}{$\begin{array}{l}{ }^{1} \text { we performed } 5 \text { iterations of the same optimization technique (algorithm Nelder-Mead) each with } 500 \text { as the } \\
\text { maximum number of iterations }\end{array}$} \\
\hline \multicolumn{6}{|c|}{2 we performed several iterations (maximal 20) until no new maximum was found } \\
\hline \multicolumn{6}{|c|}{$\begin{array}{l}31 \text { importance sampling from a uniform ball followed by } 10 \text { iterations of importance sampling from a Normal } \\
\text { distribution, sample size } 1000\end{array}$} \\
\hline \multicolumn{6}{|c|}{$\begin{array}{l}{ }^{4} \text { the final chain has a sample size of } 50000 \text {, the first } 4 \text { iterations with smaller chains were done for an optimization } \\
\text { of the jump distribution }\end{array}$} \\
\hline
\end{tabular}

Fig. 1 shows the prior marginals for the base scenario of correct priors and the corresponding results for the posterior marginals of the parameter distribution for the finite difference and the importance sampling approximation in comparison with the analogous results of the Markov chain Monte Carlo technique (Reichert and Schuwirth 2012), which samples the full posterior distribution.

As we apply the approximate technique described in section 2.3 to the log of the parameters, all posterior marginals are lognormal distributions under our simplified assumptions. The figure shows a small effect of the choice of the posterior approximation technique on the posterior marginal distri- 
$\mu$
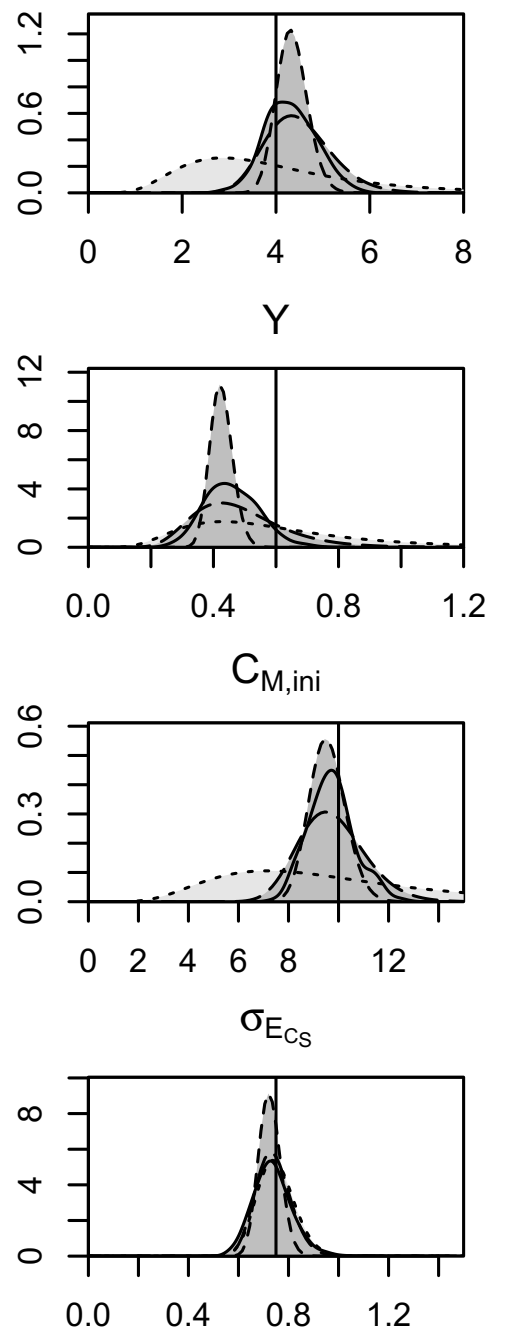

$\tau$

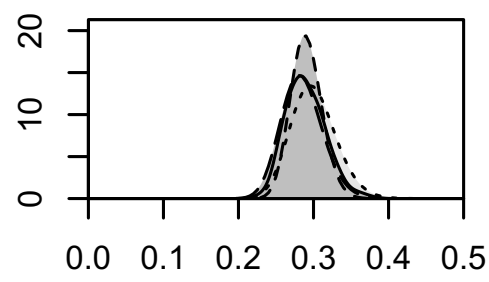

$\mathrm{K}$
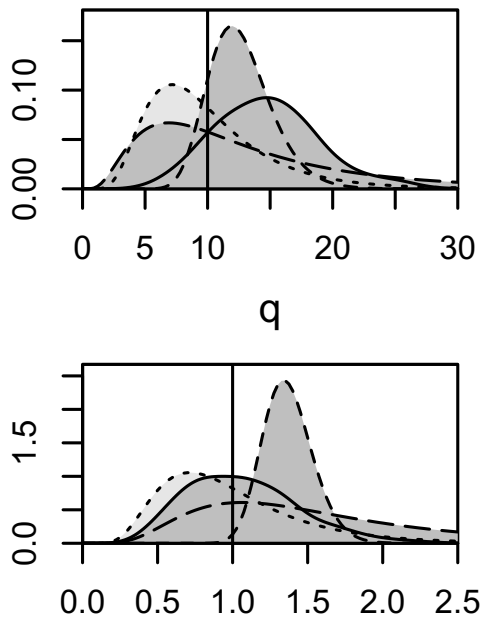

$\mathrm{C}_{\mathrm{S}, \mathrm{ini}}$

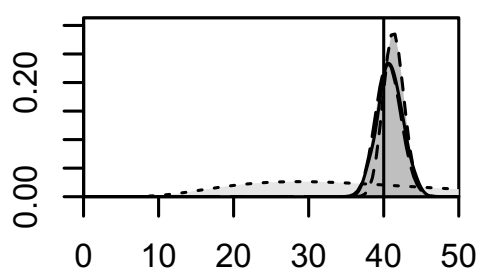

$\sigma_{\mathrm{B}_{\mathrm{M}}}$

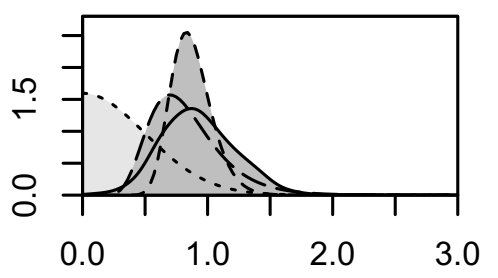

b
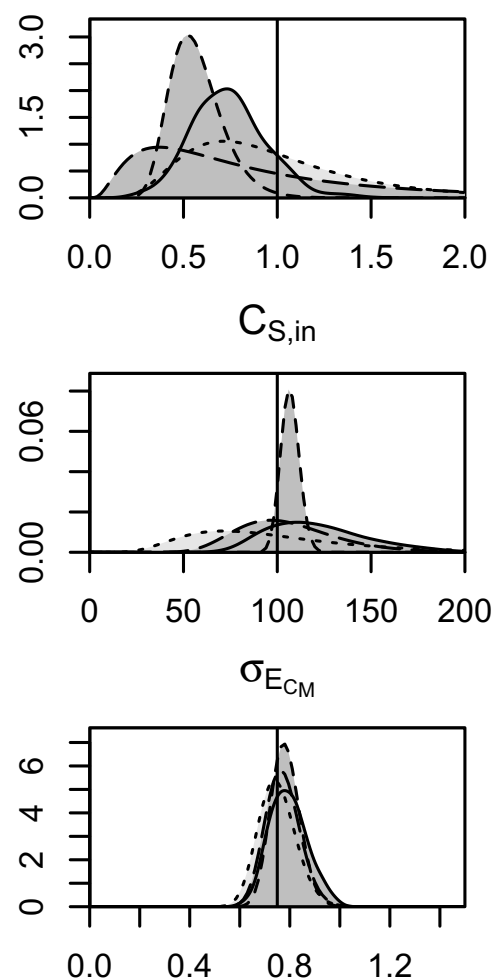

$\sigma_{\mathrm{B}_{\mathrm{CS}}}$

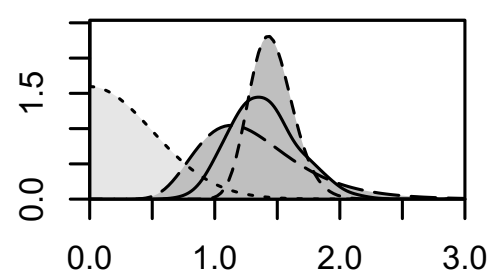

Figure 1: Prior (dotted, supported by light grey shading of the areas below the lines) and posterior (supported by dark grey shading of the areas below the lines) marginals of the model parameters for the posterior approximation by finite difference method (long-dashed), importance sampling (shortdashed) and a full Markov chain Monte Carlo method (solid) (Reichert and Schuwirth 2012). Vertical lines indicate the true values of the model parameters used for producing the synthetic data (there are no true values for the parameters of the bias). 
bution. For most parameters the results of the finite difference method show slightly wider posterior probability densities compared to the importance sampling results. Potentially, the finite difference method can be problematic as it tends to very local approximations that might underestimate the width of the posterior distribution. This does not seem to be the problem for our didactical example. Only small differences can be found for the standard deviations of the observation errors, the correlation time and the initial concentrations. The results also correspond well with the Markov chain results, although the posteriors of the two approximation methods, especially of the importance sampling technique, tend to be narrower.

Fig. 2 shows the model predictions (according to equation (22) of our knowledge of the 'true' results, $\mathbf{y}_{M}^{L_{1,2}}+\mathbf{B}_{M}^{L_{i}} \mid \mathbf{Y}_{M}^{L_{1}}$, and of observations, $\mathbf{Y}_{M}^{L_{1,2}} \mid \mathbf{Y}_{M}^{L_{1}}$, for both posterior approximation techniques shown in the different columns, again in comparison with the MCMC results (Reichert and Schuwirth 2012), for the basic prior choice.

The credibility intervals for the 'true' results, $\mathbf{y}_{M}^{L_{1,2}}+\mathbf{B}_{M}^{L_{1,2}} \mid \mathbf{Y}_{M}^{L_{1}}$, can contain a small or large fraction of future observations, depending on the available knowledge and the observation error. If the parameters would be accurately known, the credibility intervals of observations, $\mathbf{Y}_{M}^{L_{1,2}} \mid \mathbf{Y}_{M}^{L_{1}}$, should contain about $95 \%$ of future observations. However, due to the uncertainty in parameter values, they can also be considerably wider. Overall, the results demonstrate that our posterior knowledge is much more precise in times where observations are available (and used for inference) than in the extrapolation range. Further results of the posterior estimates of bias and observation error show the probabilistic division of the residuals into bias and observation error. The markers in the plots of the middle and bottom row of Fig. 2 indicate approximately normally distributed independent observation errors as required. In comparison to the results of a Markov chain Monte Carlo technique to sample from the posterior shown in the right column of Fig,2, the results of the linearized error propagation technique generally show very similar uncertainty bands, slightly narrower in the case of importance sampling. Furthermore, we see some but small effects of the choice of the posterior approximation technique on the uncertainty estimates.

Figures S1 and S2 of the supplementary data show the results of the introduced method for the choice of wrong priors, again in comparison with a Markov chain Monte Carlo approach applied to the same prior choice. Additionally to the density fit, also the finite difference technique failed in this case. Fig. S1 shows the prior and posterior marginal parameter distributions calculated by the importance sampling approximation and the MCMC method. Fig. S2 shows the results of the model simulation, the uncertainty estimation and the different contribution of bias and observation error to the uncertainty, calculated by the same methods. We see a negative effect due to the wrong prior choice, but only minor effects of the choice of the calibration and prediction method. 

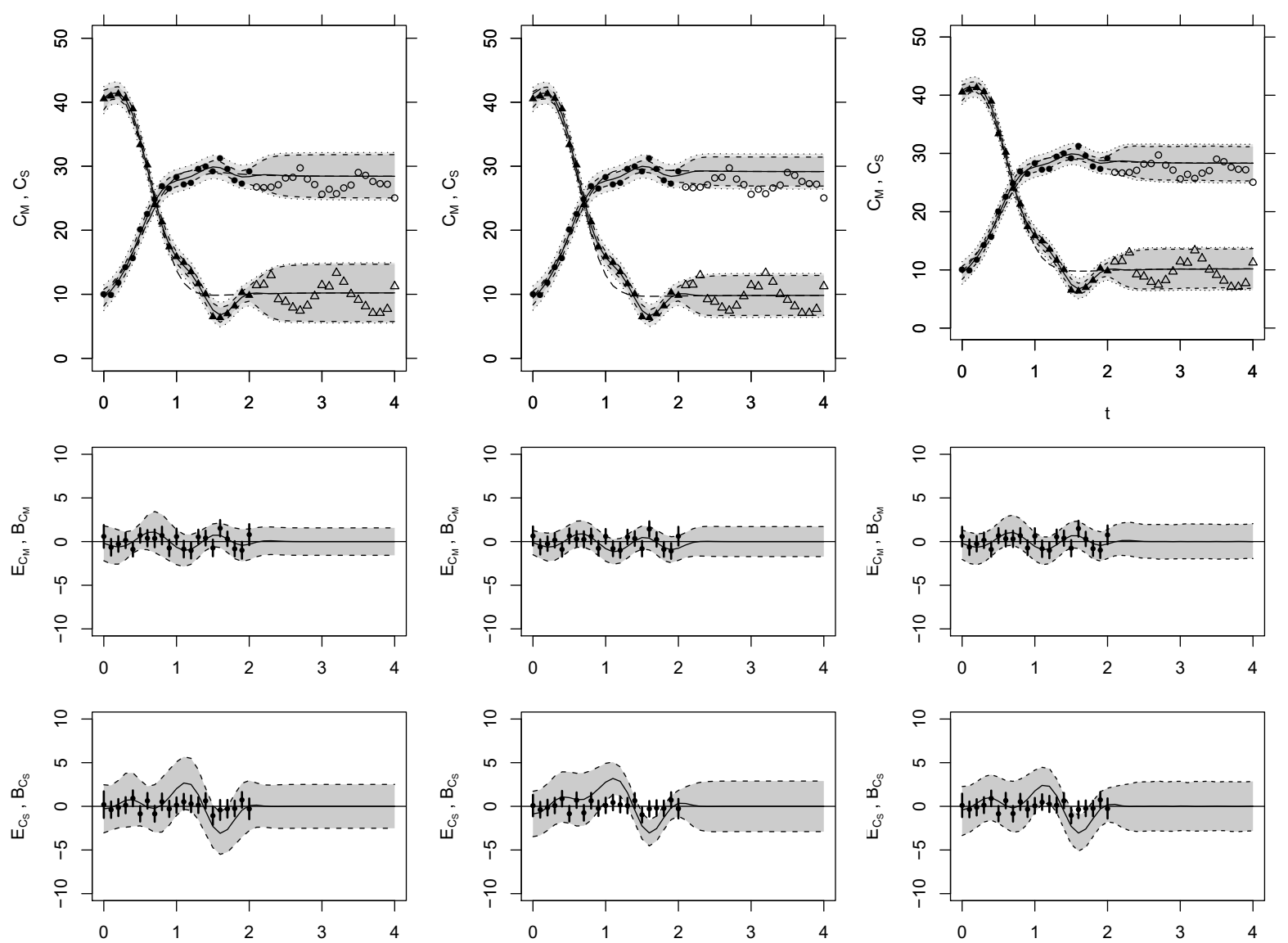

Figure 2: Results of the median and $95 \%$ credibility intervals for both variables $C_{\mathrm{M}}$ and $C_{\mathrm{S}}$ (top row; observations used for calibration are marked by solid symbols, those not used for calibration by open symbols; output of the deterministic model (long-dashed line), bias-corrected output (deterministic model plus bias; median as solid line, $95 \%$ credibility bounds as dark grey area bounded by dashed lines), and prediction for new observations (including observation error; median as solid line (same as for bias-corrected output), 95\% credibility bounds shown as dark and light grey areas bounded by dotted lines)), results of the median and $95 \%$ credibility intervals for the bias (solid line and dark grey areas bounded by dashed lines) and of the observation error (dots and vertical line segments) for the variables $C_{\mathrm{M}}$ (middle row) and $C_{\mathrm{S}}$ (bottom row). The left column shows the results for posterior approximation by finite differences, the middle column by importance sampling and the right column the results of the full MCMC method (Reichert and Schuwirth 2012). 


\section{Application to Biogeochemical and Ecological Lake Model}

In this section we give a short description of the lake model BELAMO followed by an application of the approximate calibration and prediction technique (described in the sections 2.3 and 2.4 for calibrating this model to long-term data from Lake Zurich, Switzerland.

\subsection{BELAMO: Model Description}

The Biogeochemical and Ecologial LAke MOdel (BELAMO) aims at a combined calculation of mass balances of nutrients, oxygen, organic particles, phytoplankton and zooplankton. The earliest version of BELAMO is a one-dimensional mechanistic model of nutrient and plankton dynamics developed for Lake Zurich. It models horizontally averaged substance and organism concentrations in the water column and two sediment layers as they are changing with time and the depth of the lake. For a short description of the model see Omlin et al. (2001), Mieleitner and Reichert (2006) slightly changed the one-dimensional model and analyzed its transferability to the lakes Greifensee and Walensee.

To reduce simulation time and analyze functional phytoplankton groups, the continuous vertical resolution of the model was simplified to a 4-box (epilimnion, hypolimnion and two sediment boxes) approach by Mieleitner and Reichert (2008), In these mixed boxes, concentrations of ammonium, nitrate, phosphate, oxygen, degradable and inert dead organic particles and (in the aggregated version used for this study) one group of phytoplankton and one group of zooplankton are modeled.

In the latest version of the model, the following physical processes are considered: inflow into epiand hypolimnion and outflow from the epilimnion, gas exchange with the atmosphere, mixing of all dissolved substances between epi- and hypolimnion, upwards movement of zooplankton from zones of low oxygen concentration, sedimentation of particles, advection, diffusion of dissolved substances between the pore water within the two sediment layers and the water in the hypolimnion, as well as accumulation of sediment and permanent burial.

The model considers the following biogeochemical and ecological processes: Growth, respiration and death of phyto- and zooplankton, aerobic, anoxic and anaerobic mineralization, nitrification, methane oxidation and phosphate uptake of sinking degradable and inert organic particles.

BELAMO was implemented in AQUASIM (version 2.1f, http://www.aquasim.eawag.ch), a computer program for the identification and simulation of aquatic systems (Reichert 1994 Reichert 1998). For a detailed description of the newest version of the model see Dietzel et al. (2012).

As BELAMO describes water quality and plankton dynamics, it can contribute to improving the understanding of the behavior of a lake by linking continuous-time nutrient and plankton fluxes and dynamics to (sparse) observations of concentrations only. An even more ambitious aim would be that the model supports decisions on management alternatives for environmental protection. To gain confidence in such a model application, a transparent evaluation of model performance is needed that 
takes data uncertainty, as well as model structure uncertainty, into account.

Despite the simple spatial structure of the lake model we are already faced with a complex model with 16 state variables, up to 19 processes in each box and around 70 parameters, of which at least 10 strongly influence the model results. There is not much prior knowledge available for several of these parameters. Examples are the mineralization rates of aerobic, anoxic and anaerobic mineralization processes that represent a combination of bacterial densities and their growth rates. Only indirect information is available through their influence on observed concentrations of oxygen, nitrate and organic material in the water column of the lake.

On the one hand, the complexity of the model results in simulation times of at least 1 minute per year. This makes usual Bayesian numerical techniques, such as Markov chain Monte Carlo simulations, difficult to apply for long-term simulations. On the other hand, the coarse description of plankton communities with an aggregation into one group of phyto- and one group of zooplankton, as well as the spatial aggregation of the whole lake depth into two boxes and process simplifications, cause significant biases. This is particularly the case as this model closes mass balances by explicitly considering mineralization in the sediment. This couples the model variables much stronger than it is the case in more widespread lake models that use sediment fluxes as independent parameters (Mooij et al. 2010). Without making the model even more complex, this bias can not be significantly reduced by extending the model structure. Furthermore, as we have model outputs and data for evaluation of both nutrients and plankton, we are confronted with a multi-objective calibration problem. Manual calibrations showed the difficulty of calibrating the model evenly well for all output variables during the whole simulation time. Because of these problems, an uncertainty estimation, as the one described above, became necessary. As a first calibration step, the model was applied to long-term observations of Lake Zurich. As BELAMO should describe the processes in lakes as universally as possible, we tend to a joint calibration of the model parameters for lakes of different trophic states but similar climate and mixing conditions. In Dietzel et al. (2012) we show the results of a joint calibration of three Swiss lakes with different trophic states with the suggested calibration and prediction technique.

\subsection{Study Area}

Lake Zurich is located in the north-eastern part of the Swiss plateau. At its deepest point the lake is $136 m$ deep. We apply the model to the lower part of Lake Zurich only, which has a length of around $28 \mathrm{~km}$. The catchment of Lake Zurich is mainly influenced by urban areas and less by agriculture. As measured by prevailing phosphorus concentrations, Lake Zurich is a mesotrophic lake. For a detailed description of the main lake attributes see Mieleitner and Reichert (2006), for a location map see 


\subsection{Data}

Monthly measured profiles of physical, chemical and biological variables for Lake Zurich were obtained from 1976 to 2005 (zooplankton only since 1985). These data were collected by the Water Supply Authority of Zurich (Wasserversorgung Zürich, WVZ). Information on inflowing rivers and contributing waste water treatment plants (physical and chemical parameters) and meteorological data were received from federal and cantonal agencies and from technical reports.

Data from input, concentrations in the lake and meteorological forcing were converted to AQUASIM format. Data compilation was done with S-Plus. Accounting for the availability of input and validation data we accomplished model calibrations with the data from 1976 up to and including 1995. Hence, our observation layout $L_{1}$ indicates combinations of monthly sampling dates, the five calibrated output variables phytoplankton, zooplankton, nitrate, oxygen and phosphate and the two compartments epilimnion and hypolimnion. Simulations and data of the following 10 years up to the end of 2005 were used for model validation. The monthly sampling dates of these years in combination with each of the variables and compartments compose the prediction layout $L_{2}$. For a detailed description of the available data and its compilation see Dietzel et al. (2012)

We assume that observation errors as well as bias are smaller for smaller concentrations of chemical and biological variables. To account for this heteroscedasticity of the data, we conducted a BoxCox transformation of data and model results as described in section 2.2 (Box and Cox 1964). For the application to BELAMO, the transformation parameters $\lambda_{1}$ and $\lambda_{2}$ were chosen as 0.5 and 0 , respectively.

\subsection{Prior Distribution}

The parameters included in the parameter estimation procedure comprise a selection of model parameters and the standard deviations of the observation errors and the bias for all considered output variables.

Concerning the model parameters of the deterministic model, $\boldsymbol{\theta}$, we concentrated on $k_{g r o, A L G, T_{0}, I_{0}}$, the growth rate of phytoplankton at reference temperature and reference light conditions, $k_{\text {death }, A L G, T_{0}}$, the death rate of phytoplankton at reference temperature, $k_{\text {gro,ZOO, } T_{0}}$ and $k_{\text {death,ZOO,T}}$, the growth and death rate of zooplankton at reference temperature. Furthermore, the aerobic, anaerobic and anoxic mineralization rates at reference temperature, $k_{\text {miner,aero,sed, } T_{0}}, k_{\text {miner,anae,sed, } T_{0}}$ and $k_{\text {miner,anox,sed,T }}$ are considered for calibration, as well as the threshold concentration of phytoplankton when zooplankton feeding switches from quadratic to Monod-type limitation, $K_{F e e d}$, and $f_{X_{I}}$,rivers , the fraction of inert organic particles of allochthonous input. We chose these parameters because of uncertain knowledge about their value in nature. Good estimates are neither available for the average growth and death rates of the aggregated phyto- and zooplankton communities, nor for the mineralization rates that 
Table 2: Prior marginals for the calibrated parameters of $\boldsymbol{\theta}$ of the deterministic model.

\begin{tabular}{l|c|l|c|c} 
Name & Unit & Distribution & Mean & StDev \\
\hline$k_{\text {gro }, A L G, T_{0}, I_{0}}$ & $\mathrm{~d}^{-1}$ & Lognormal & 1.79 & 0.5 \\
$k_{\text {death }, A L G, T_{0}}$ & $\mathrm{~d}^{-1}$ & Lognormal & 0.0721 & 0.05 \\
$k_{\text {gro }, Z O O, T_{0}}$ & $\mathrm{~d}^{-1}$ & Lognormal & 0.397 & 0.25 \\
$k_{\text {death }, Z O O, T_{0}, z h}$ & $\mathrm{~d}^{-1}$ & Lognormal & 0.0675 & 0.05 \\
$K_{\text {Feed }}$ & $\mathrm{gDM} \mathrm{m}^{-3}$ & Lognormal & 0.295 & 0.3 \\
$f_{X_{I}, \text { rivers }}$ (transformed) & - & Normal & 2 & 1 \\
$k_{\text {miner,aero, }, \text { sed }, T_{0}, z h}$ & $\mathrm{~d}^{-1}$ & Lognormal & 0.295 & 0.3 \\
$k_{\text {miner,anae }, \text { sed }, T_{0}, z h}$ & $\mathrm{~d}^{-1}$ & Lognormal & 0.295 & 0.3 \\
$k_{\text {miner,anox }, \text { sed }, T_{0}, z h}$ & $\mathrm{~d}^{-1}$ & Lognormal & 0.295 & 0.3
\end{tabular}

depend on the composition and abundances of the bacterial communities. Moreover, these parameters are the most influential ones, as they describe main aspects of the plankton kinetics and control the nutrient dynamics to some extent. For a more detailed description of the parameter choice and the results of a sensitivity analysis we refer to Dietzel et al. (2012) The prior marginals, reflecting our vague prior knowledge about the parameter values, are given in Table 2 Except for $f_{X_{I}, \text { rivers }}$, lognormal distributions, which avoid biologically impossible negative values, were chosen as prior marginals for the model parameters with a mode representing our best estimate of the parameter value. As $f_{X_{I}, \text { rivers }}$ defines a fraction, this parameter is restricted to values between 0 and 1 . We performed a transformation of this parameter by means of an arctangent function. For the prior marginal of the transformed parameter, a Normal distribution was assumed. The prior knowledge of all calibrated model parameters was formulated by relatively wide marginal distributions accounting for the high parameter uncertainty. The assumed values of the remaining parameters of $\boldsymbol{\theta}$ can be found in Omlin et al. (2001), Mieleitner and Reichert (2008) and Dietzel et al. (2012) The results of the calibration and prediction method depend only slightly on the choice of these remaining, not calibrated model parameters, as the most influential and less known parameters were chosen for calibration.

Because the Box-Cox transformation accounts for heteroscedasticity, we can choose the same standard deviation of the observation error over the whole range of (Box-Cox transformed) measured concentrations for each output variable. The same is true for the bias. We assumed lognormal priors for the standard deviations of the observation errors representing the parameter vector $\boldsymbol{\psi}$. These values are listed in Table 3 in Box-Cox transformed units. The chosen means reflect our knowledge that, due to spatial heterogeneity ("patchiness") and higher measurement error, plankton data are prone to larger observation errors than nutrient data. The values were chosen by assuming a smaller relative error compared to the typical (average) concentrations of the different variables and transforming the respective absolute values which have the same units as the measured variables by a Box-Cox 
Table 3: Prior marginals for the parameters $\boldsymbol{\psi}$ of the error model (on a Box-Cox transformed scale).

\begin{tabular}{l|c|l|c|c} 
Name & Unit & Distribution & Mean & StDev \\
\hline$\sigma_{\mathrm{E}, \mathrm{ALG}}$ & $\mathrm{gWM}^{1 / 2} \mathrm{~m}^{-3 / 2}$ & Lognormal & 0.07 & 0.02 \\
$\sigma_{\mathrm{E}, \mathrm{ZOO}}$ & $\mathrm{gWM}^{1 / 2} \mathrm{~m}^{-3 / 2}$ & Lognormal & 0.08 & 0.02 \\
$\sigma_{\mathrm{E}, \mathrm{NO} 3}$ & $\mathrm{gN}^{1 / 2} \mathrm{~m}^{-3 / 2}$ & Lognormal & 0.022 & 0.002 \\
$\sigma_{\mathrm{E}, \mathrm{O} 2}$ & $\mathrm{gO}^{1 / 2} \mathrm{~m}^{-3 / 2}$ & Lognormal & 0.11 & 0.01 \\
$\sigma_{\mathrm{E}, \mathrm{HPO} 4}$ & $\mathrm{gP}^{1 / 2} \mathrm{~m}^{-3 / 2}$ & Lognormal & 0.007 & 0.0002
\end{tabular}

Table 4: Prior marginals for the calibrated parameters of $\boldsymbol{\xi}$ of the bias (on a Box-Cox transformed scale).

\begin{tabular}{l|c|c|c} 
Name & Unit & Distribution & Mean \\
\hline$\sigma_{\mathrm{B}, \mathrm{ALG}}$ & $\mathrm{gWM}^{1 / 2} \mathrm{~m}^{-3 / 2}$ & Exponential & 0.3 \\
$\sigma_{\mathrm{B}, \mathrm{ZOO}}$ & $\mathrm{gWM}^{1 / 2} \mathrm{~m}^{-3 / 2}$ & Exponential & 0.4 \\
$\sigma_{\mathrm{B}, \mathrm{NO} 3}$ & $\mathrm{gN}^{1 / 2} \mathrm{~m}^{-3 / 2}$ & Exponential & 0.11 \\
$\sigma_{\mathrm{B}, \mathrm{O} 2}$ & $\mathrm{gO}^{1 / 2} \mathrm{~m}^{-3 / 2}$ & Exponential & 0.5 \\
$\sigma_{\mathrm{B}, \mathrm{HPO} 4}$ & $\mathrm{gP}^{1 / 2} \mathrm{~m}^{-3 / 2}$ & Exponential & 0.035
\end{tabular}

transformation to the shown means.

Last, Table 4 contains the priors for the standard deviations of the bias in Box-Cox transformed units. We chose exponential distributions for which the probability density increases with decreasing value of the standard deviation. This reflects our desire to avoid bias if possible. The differences between the priors for the different output variables indicate rather a relative weighting than an assumption of the absolute magnitude of the bias, they coarsely represent around $25 \%$ bias (compared to typical concentrations) for the plankton and around $15 \%$ for the chemical variables. In general, we assume the bias to be larger than the observation error. The parameter $\beta$ that determines the correlation structure was fixed to $0.00005 \mathrm{~d}^{-2}$, representing a correlation time of about four months. As this parameter would be difficult to identify and is coarsely known for a lake strongly affected by annual mixing processes, it was not included in the calibration procedure. The standard deviations of the bias and the parameter $\beta$ represent the parameter vector $\boldsymbol{\xi}$.

For the parameter estimation, the latest version of UNCSIM Reichert (2005), a program package for statistical inference, identifiability analysis and uncertainty analysis, was used by coupling with AQUASIM. Further calculations for the prediction layout and the approximation of the posterior distribution of model results at its maximum for estimation of the prediction uncertainty were done with the statistics software R (http://www.r-project.org/). 


\subsection{Results and Discussion}

A calibration period of 20 years (ending in 1995) was chosen, the description of the observations of those years served as layout $L_{1}$. The prediction uncertainty was estimated for the validation period of the following 10 years defined by $L_{2}$. Observations are also available for layout $L_{2}$. They were not used for calibration, but serve as validation data for comparison with model results. The deterministic model function, $\mathbf{y}_{M}^{L}(\boldsymbol{\theta})$, consists of model outputs for the different variables oxygen, phosphate, nitrate, phytoplankton and zooplankton in the epilimnion and hypolimnion at discrete simulation times specified by the same layouts.

Inference was again done by applying all three options for deriving a Normal distribution as an approximation to the posterior at its maximum described in section 2.3.1 The maximum was obtained before by applying the shuffled complex evolution algorithm (Duan et al. 1993) with UNCSIM. Application of the finite difference algorithm was not successful as we could not achieve that all incremented simulations led to a smaller posterior than the base simulation found by the optimization algorithm. Due to the computational demand we could not redo the analysis very frequently at the newly found maximum. The complex shape of the posterior, the inaccuracy of the optimization algorithm, and the small increments required for the finite difference approximation may all have contributed to this problem. In contrast to the didactical example, for this application, it was possible to fit a multivariate Normal distribution by density fitting (we chose the simplified approach described in section 2.3.1 that keeps the mean at the parameter values corresponding to the maximal posterior pdf value). Finally, we did importance sampling from a uniform distribution in a ball (large enough to approximately cover the whole posterior) which was refined by an importance sampling using the Normal distribution with mean and variance-covariance matrix from the first importance sampling as a sampling distribution. Table 5 summarizes the computational demand of the applied techniques.

Table 5: Number of simulation runs needed for the optimization and the different approximation techniques for the application case of BELAMO. All numbers refer to the simulation runs actually performed for the results shown herein.

\begin{tabular}{l||c|c|c|c|c} 
Application Case & Optimization & $\begin{array}{c}\text { Finite } \\
\text { Difference }\end{array}$ & $\begin{array}{c}\text { Density } \\
\text { Fitting }\end{array}$ & $\begin{array}{c}\text { Importance } \\
\text { Sampling }\end{array}$ & MCMC \\
\hline BELAMO & $\sim 4000^{[1]}$ & $722(\cdot \mathrm{x})^{[2]}$ & 600 & $2 \cdot 200^{[3]}$ & -
\end{tabular}

some preliminary optimization runs for parameter estimation were done before

we performed several iterations but still new "maxima" were found

1 importance sampling from a uniform ball followed by 1 importance sampling from a Normal

distribution, sample size 200

The comparison of prior and posterior marginal distributions of the model parameters and the parameters of the error model are shown for both successful approaches in Fig. 3 The results presented in this figure show a dependence on the chosen approximation technique, but for most parameters 
the marginals are similar. For some parameters the position of the maximum depends on the chosen approximation technique, for others just the width of the distribution. When applying the technique of importance sampling from a uniform distribution in a ball around the maximum, it must be guaranteed that the ball is large enough to cover the relevant part of the posterior to get unbiased results. This can be difficult as the efficiency of the procedure decreases with increasing radius of the ball. The method is more robust when followed by an importance sampling from the resulting normal distribution as shown herein. For some parameters the differences between prior and posterior distribution are relatively large. Due to unknown bacterial densities, the mineralization rates are not well known and wide priors were chosen. The shift to the posterior shows that we learn something about the values of these parameters. For the standard deviations of the observation errors, the deviations between priors and posteriors show that the priors might have been chosen slightly too small or too narrow or that some of the natural variability is "interpreted" as observation error by the model. For the standard deviations of the bias, the posterior distributions show how few bias is possible, which is the only requirement formulated by the exponential priors, here the shift is not an indication for a prior-data conflict.

The long-dashed lines in Figs. 4- 6 show the simulation results of BELAMO for the fitted output variables at the maximum of the posterior for both the calibration and the prediction time. They represent the median of the distribution of model results, $\mathbf{y}_{M}^{L_{1,2}}$, due to the posterior distribution of model parameters, $\boldsymbol{\theta}$, conditional on the model results of layout $L_{1}$ without contribution of the bias. The dark grey areas in Figs. $4-6$ depict our knowledge about the true state of the system $\left(\mathbf{y}_{M}^{L_{1,2}}+\mathbf{B}_{M}^{L_{1,2}}\right.$, model results plus bias without measurement error $)$, the light grey areas that of potential new observations $\left(\mathbf{Y}_{M}^{L_{1,2}}\right)$. In the first part, observations were used to enhance our knowledge. In the second part, observations were not used for inference and thus our results can be seen as a prediction of the "future" state of the system. Observations within layout $L_{2}$ are shown for validation of the predictive power of the model. Depending on the correlation time, the gained information about the bias for layout $L_{1}$ gives information also for the beginning of the validation time. After the correlation between the bias of layout $L_{1}$ and $L_{2}$ decreased to zero, the median of $\mathbf{y}_{M}^{L_{1,2}}+\mathbf{B}_{M}^{L_{1,2}} \mid \mathbf{Y}_{M}^{L_{1}}$ (solid line in Fig. 4-6. equals the median of $\mathbf{y}_{M}^{L_{1,2}}$ (long-dashed line). The shown results represent the results from the density fitting technique. However, the results from the importance sampling technique, conducted for this case study, show large similarities.

The uncertainty due to parameter uncertainty and bias (width of the dark grey band in Figs. 4- 6 is generally quite narrow during the calibration period (where the data points constrain the predictions) and increases considerably during the prediction phase. For all chemicals (dissolved oxygen, nitrate and phosphate) the observation error can be neglected in the prediction period compared to the bias, whereas there is still considerable observation error for the biological variables. As mentioned before, 

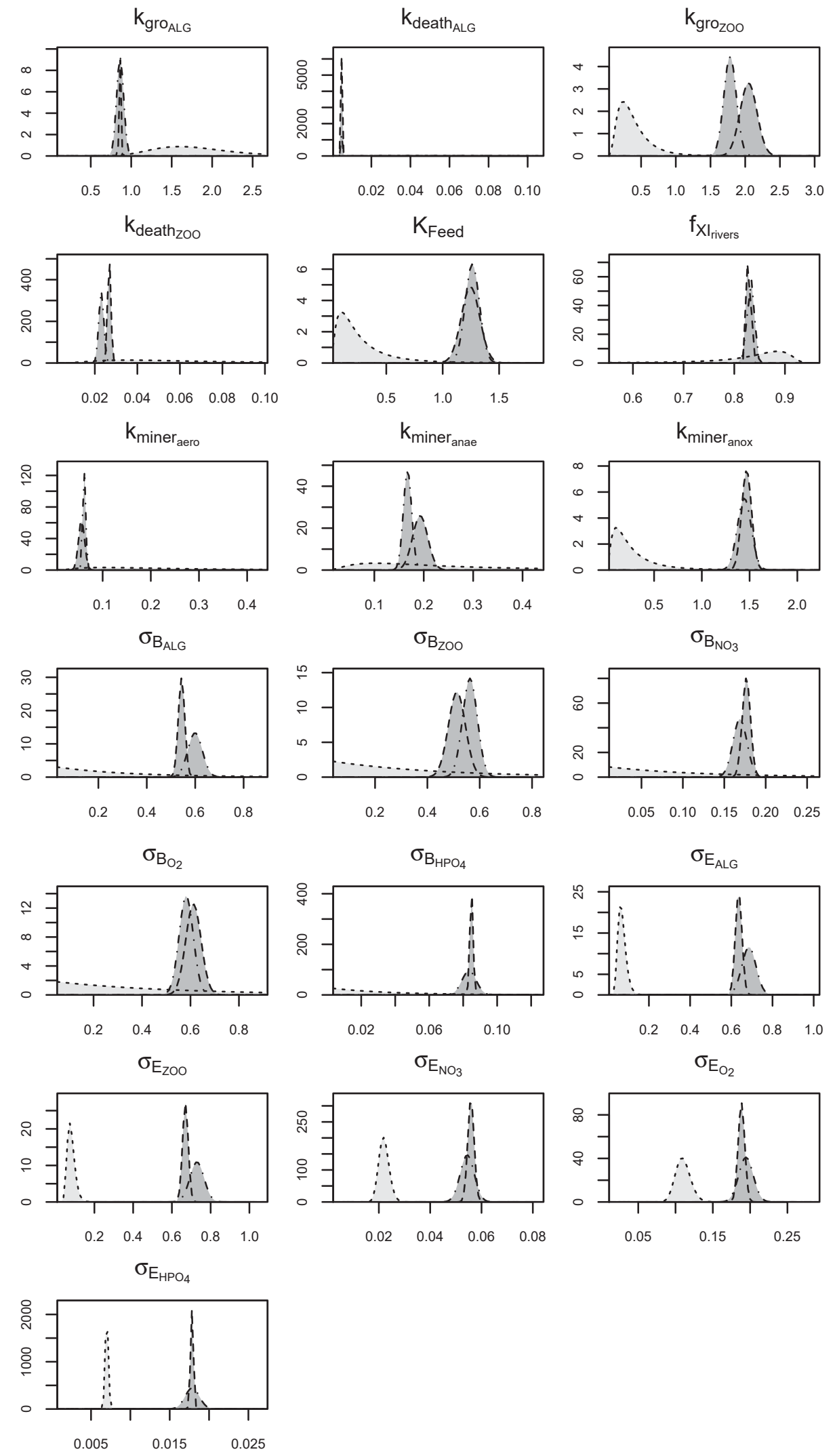

Figure 3: Prior (dotted, supported by light grey shading of the areas below the lines) and posterior (supported by dark grey shading of the areas below the lines) marginals of the model parameters from the posterior approximation by combined ball/normal importance sampling (dashed) and a density fit (dash-dotted). 


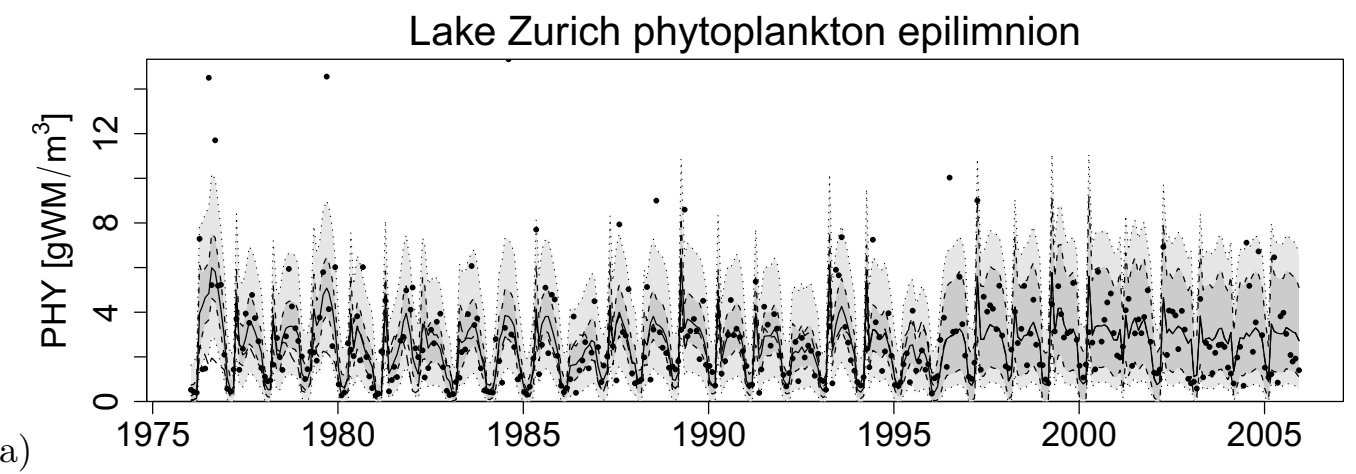

$(\mathrm{a})$

Lake Zurich phytoplankton hypolimnion

(b)

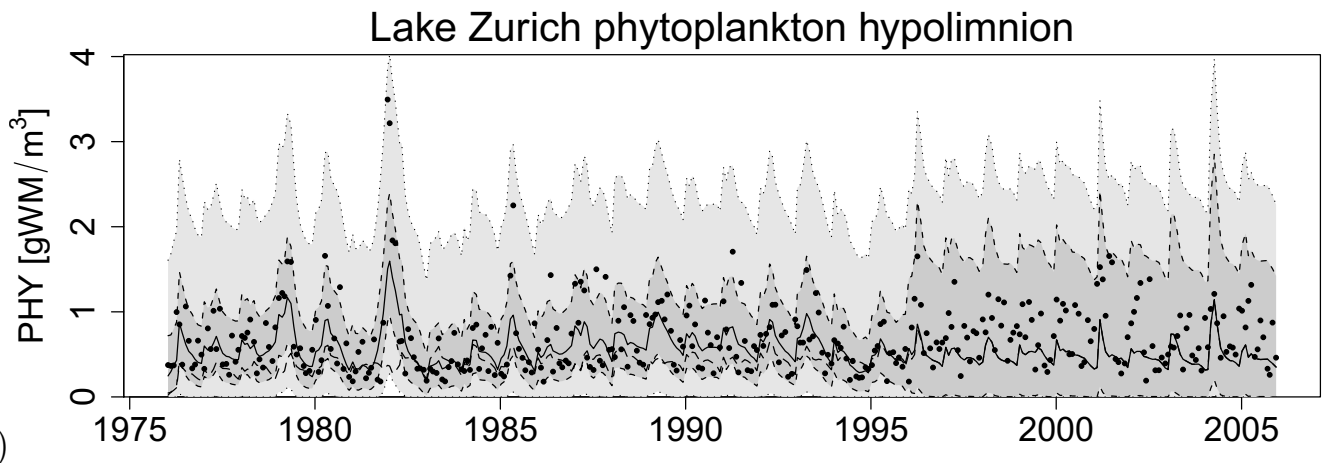

Lake Zurich zooplankton epilimnion

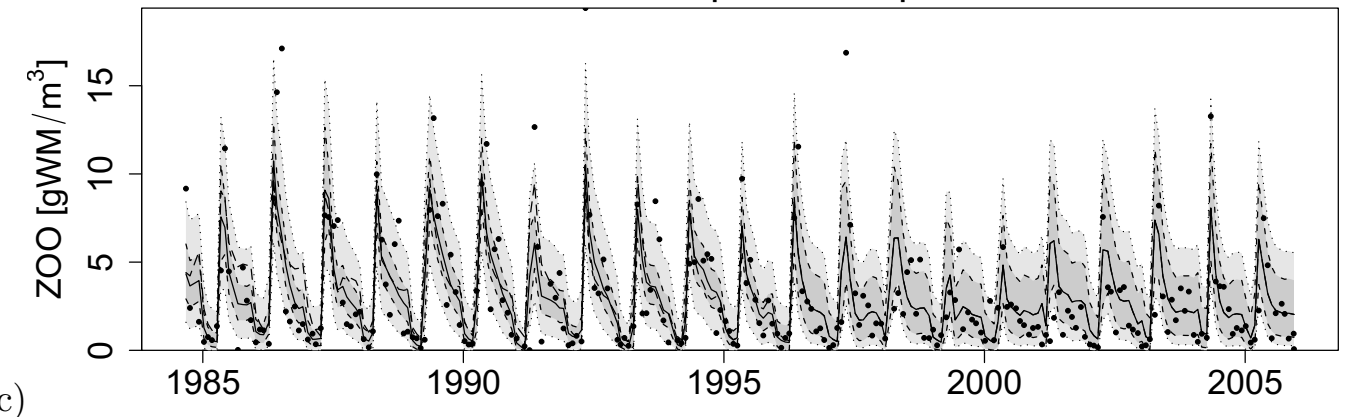

(c)

Lake Zurich zooplankton hypolimnion

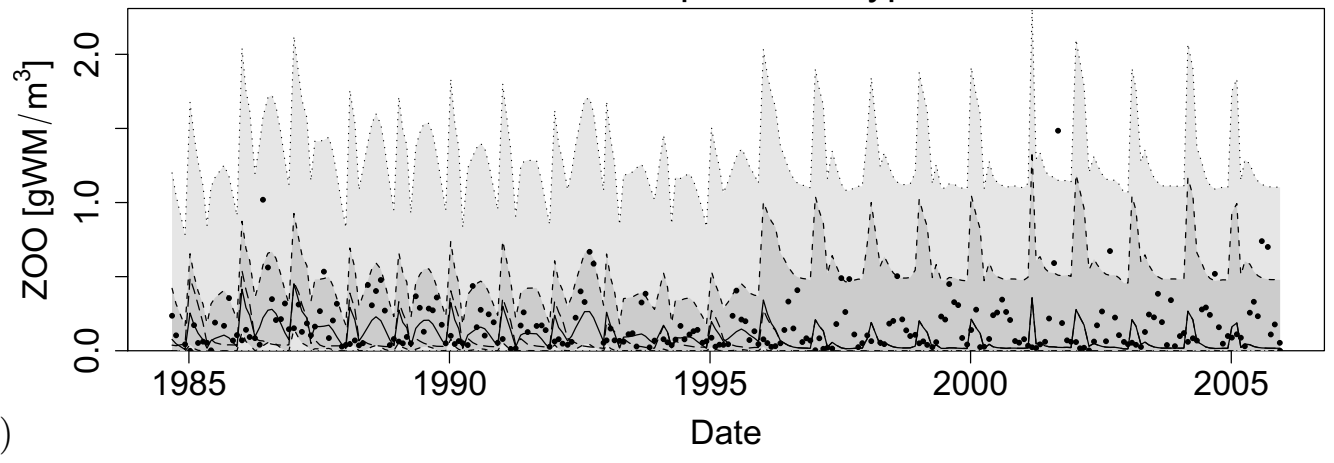

Figure 4: Phytoplankton ((a),(b)) and zooplankton $((\mathrm{c}),(\mathrm{d}))$ concentrations in the epilimnion ((a),(c)) and hypolimnion $((\mathrm{b}),(\mathrm{d}))$ of Lake Zurich. Data points (markers), output of the deterministic model (long-dashed), median (solid) and 95\% credibility bounds (dark grey area with dashed boundaries) of bias-corrected output and median (solid; same as for bias-corrected output) and $95 \%$ credibility bounds (dark and light grey areas with dotted boundaries) of predictions of new observations (including observation error) for the whole simulation time. 


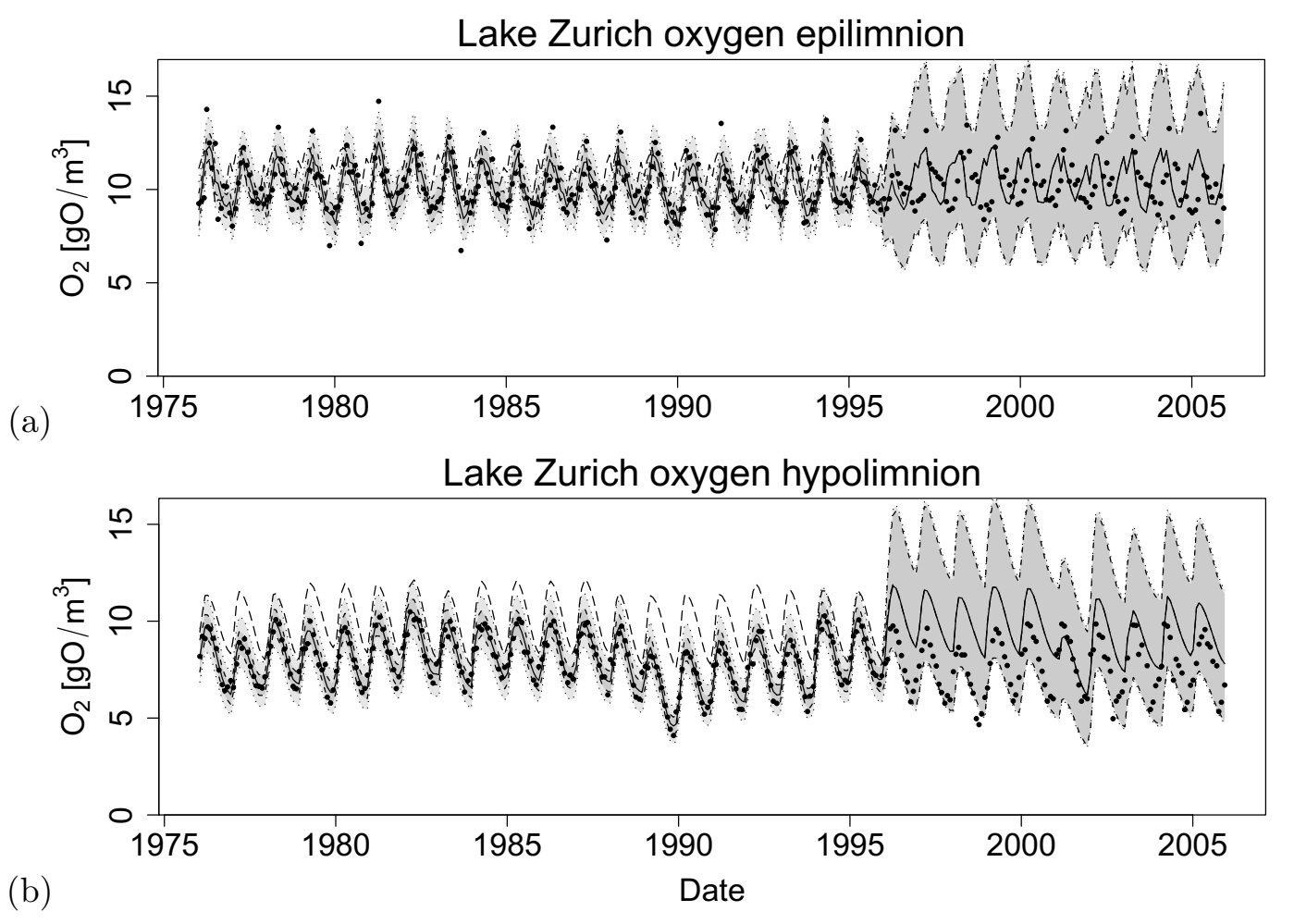

Figure 5: Oxygen concentrations in the epilimnion (a) and hypolimnion (b) of Lake Zurich. Data points (markers), output of the deterministic model (long-dashed), median (solid) and $95 \%$ credibility bounds (dark grey area with dashed boundaries) of bias-corrected output and median (solid; same as for bias-corrected output) and $95 \%$ credibility bounds (dark and light grey areas with dotted boundaries) of predictions of new observations (including observation error) for the whole simulation time. 


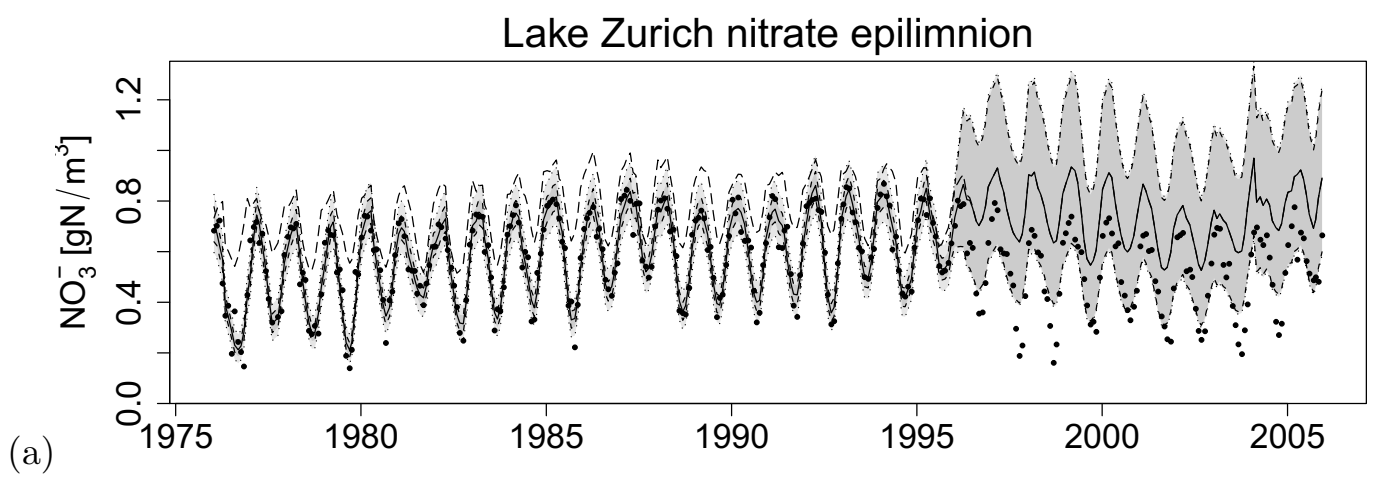

(a)

Lake Zurich nitrate hypolimnion

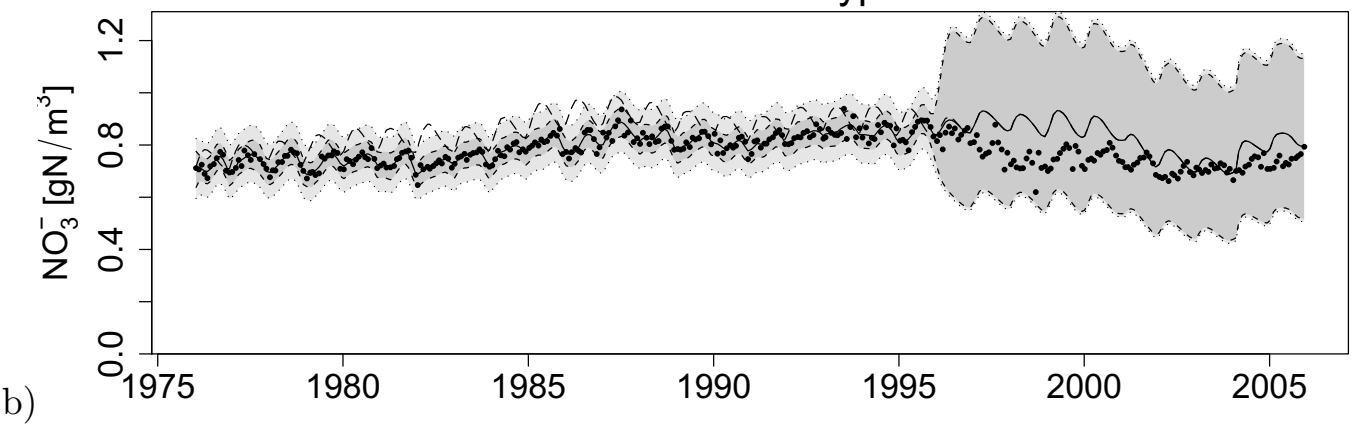

Lake Zurich phosphate epilimnion

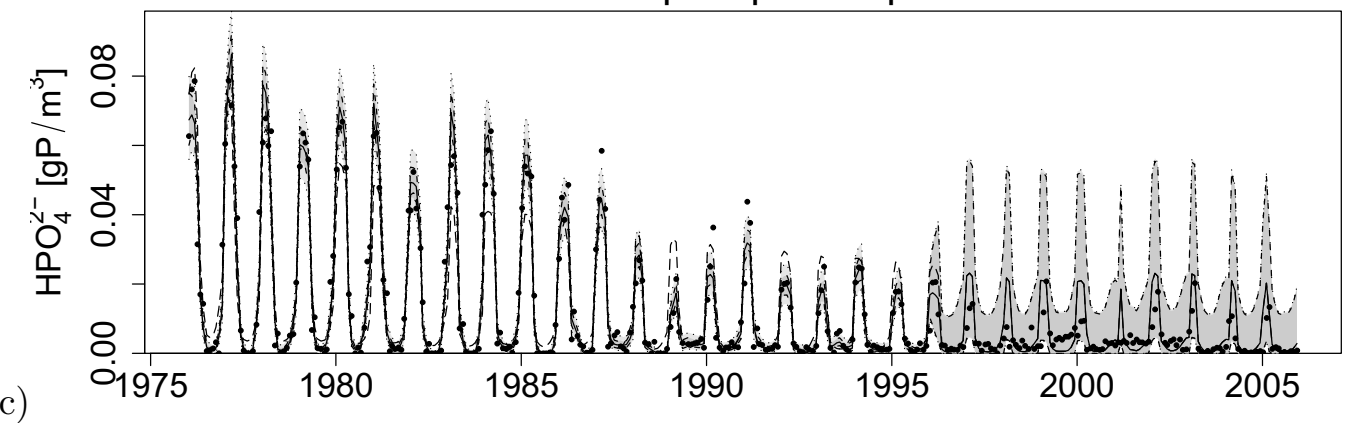

(c)

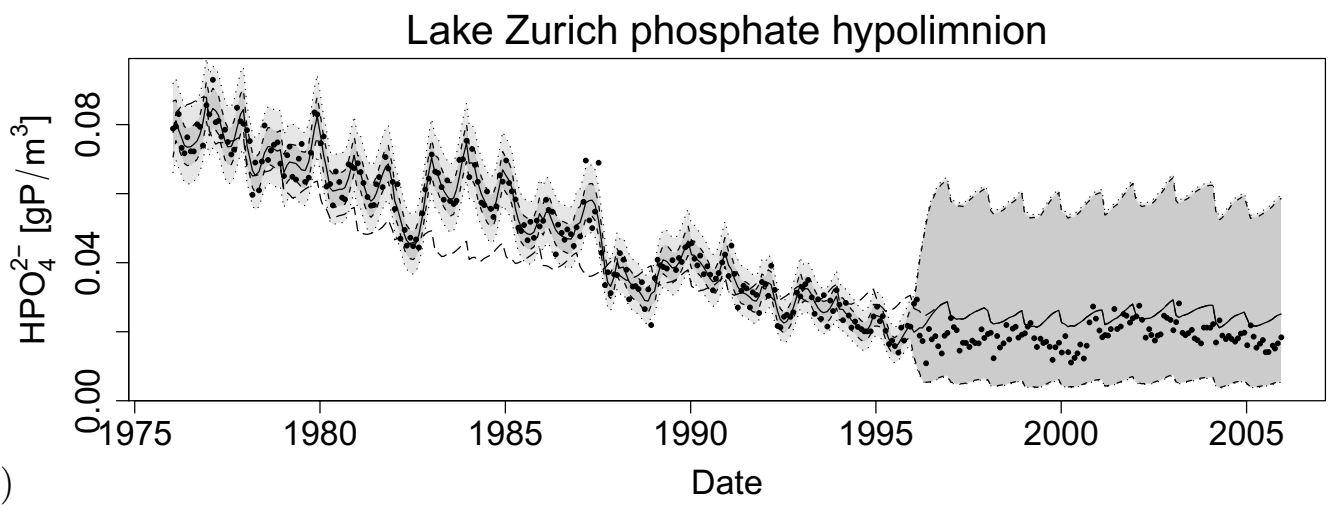

Figure 6: Nitrate $((\mathrm{a}),(\mathrm{b}))$ and phosphate $((\mathrm{c}),(\mathrm{d}))$ concentrations in the epilimnion $((\mathrm{a}),(\mathrm{c}))$ and hypolimnion ((b),(d)) of Lake Zurich. Data points (markers), output of the deterministic model (long-dashed), median (solid) and 95\% credibility bounds (dark grey area with dashed boundaries) of bias-corrected output and median (solid; same as for bias-corrected output) and $95 \%$ credibility bounds (dark and light grey areas with dotted boundaries) of predictions of new observations (including observation error) for the whole simulation time. 
this reflects the larger sampling error due to "patchiness" and the larger measurement error of the biological variables compared to the chemical variables. Generally, a comparison of the results indicates that annual patterns of both phyto- and zooplankton seem to be most difficult to be represented by the model. The deviations most likely result from simplifications by spatial and functional aggregation in the model. These simplifications are less realistic for biological than for chemical variables.

The good representation of data by the model results plus bias compared to the poorer deterministic model results demonstrates the large contribution of bias in the model results. A more detailed insight into the importance of bias for the different output variables can be given by Fig. 7 which shows the median and the $95 \%$ credibility interval of the bias and the observation error of all output variables. All graphs indicate that the structural error of the model is mainly captured by our bias description, whereas the measurement error seems to be normally distributed and independent as required.

(a)

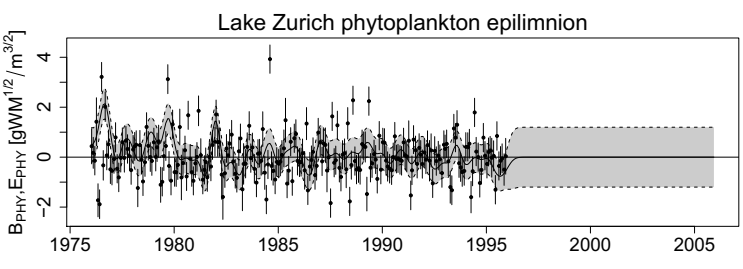

(c)

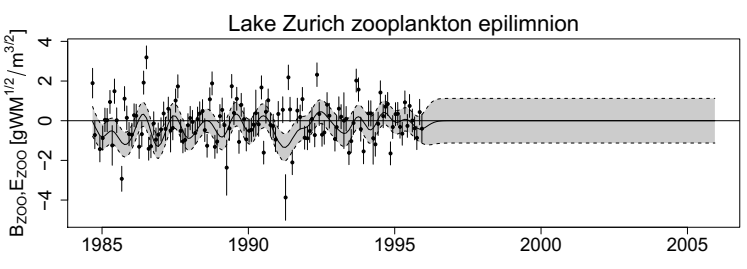

Lake Zurich oxygen epilimnion

(e)

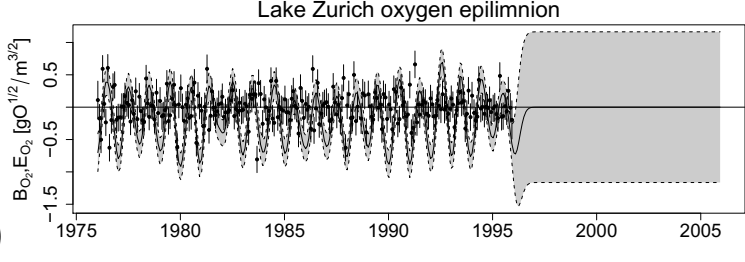

Lake Zurich nitrate epilimnion

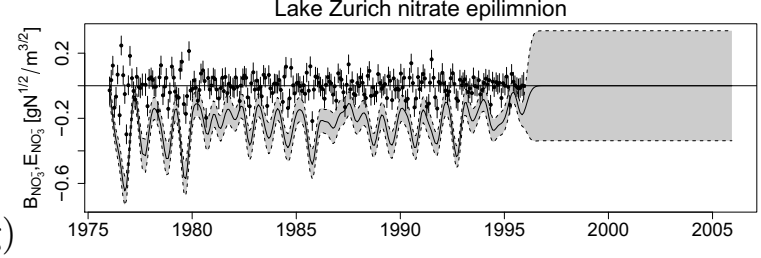

(g)

(i)

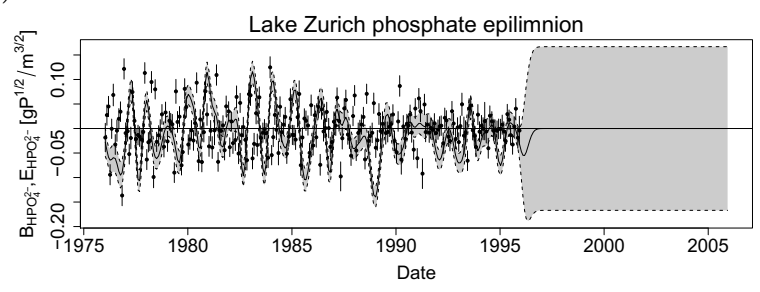

(b)
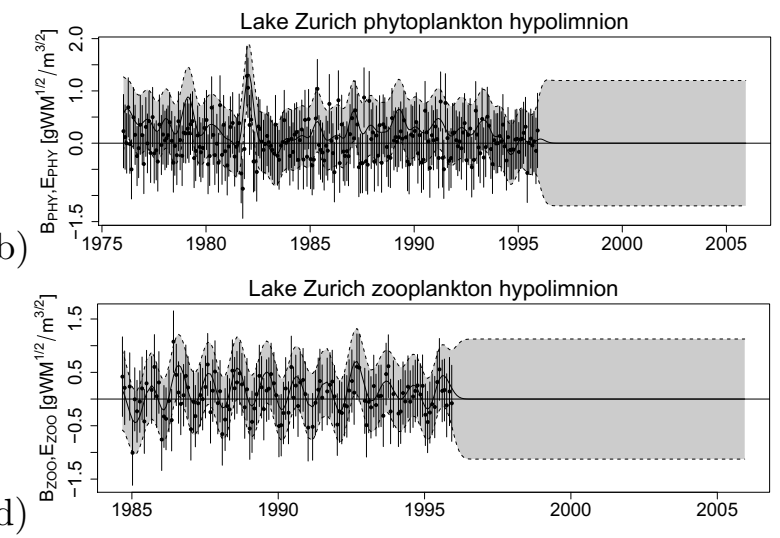

(d)

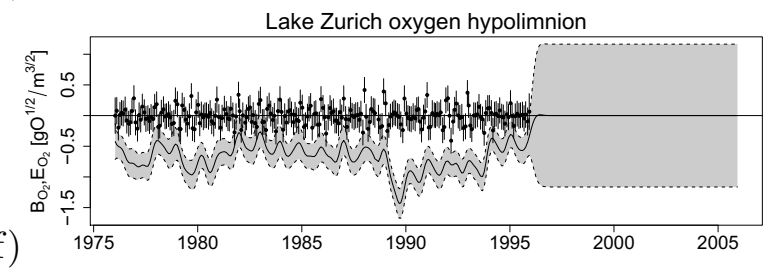

Lake Zurich nitrate hypolimnion

(h)
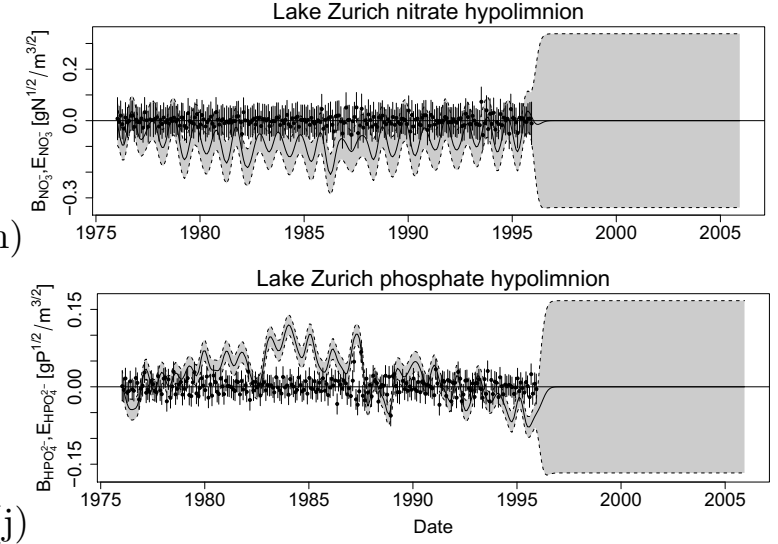

Figure 7: Median and $95 \%$ credibility interval of posterior estimate of bias (solid line and shaded area bounded by dashed lines) and median posterior estimate and uncertainty of observation error (markers and vertical lines) of observed output variables in Lake Zurich. The units refer to the units of respective output variables on a Box-Cox transformed scale.

In general, the simulation and uncertainty results show the ability of the model to describe the 
most important processes in the studied lake in a meaningful way. But the deviations between data and model results also demonstrate the difficulty of a multi-objective calibration of different output variables over a long-term period. The large uncertainties indicate the limited prediction ability of such a deterministic model.

\section{Conclusions}

Previous experiences in the literature, the technical development in this paper and the practical experiences with a didactical example and an application to a lake water quality model in this paper lead us to the following conclusions:

1. The Normal approximation to the posterior at its maximum and the linearized uncertainty propagation technique developed in this paper for the bias description technique described above considerably reduces the computational requirements of the method introduced by Reichert and Schuwirth (2012), Starting with the same optimization procedure (that is hard to avoid for any model calibration procedure), the tens of thousands of simulation runs for the Markov chain are replaced by a few hundred (or few thousand) simulation runs required to achieve the Normal approximation to the maximum of the posterior and the error propagation. This makes the approximate version of the important technique mentioned above applicable to a much larger class of computationally demanding simulation models, specifically to large group of environmental models.

2. The shape of the posterior is often complicated (Gupta et al. 2003) which can make its approximation by a Normal distribution challenging. We proposed three options to get such an approximation: A finite difference approximation at the maximum, a fit of a Normal density to a parameter sample with corresponding posterior values, and derivation of the mean and variance-covariance matrix from such a sample using importance sampling. The finite difference approach may be difficult to apply due to local maxima of the posterior density and an inaccurate determination of the maximum by the numerical maximization algorithm. The density fitting approach may have difficulties due to the large number of parameters and the constraints they have to fulfill. Indeed, each of these approaches failed in one of the two case studies of this paper. The importance sampling approach seems to be more robust and could be applied for both cases.

3. The experiences with the lake water quality model BELAMO indicate strong difficulties in calibration. Unlike most other lake water quality models (Mooij et al. 2010), BELAMO (Omlin et al. 2001; Mieleitner and Reichert 2006, Mieleitner and Reichert 2008) uses closed material balances by including a sediment compartment with two zones and coupling diagenetic processes 
in the sediment to the nutrient dynamics of the water column. This leads to a much stronger coupling between different model variables and makes unbiased calibration much more difficult than with independent parameters for release of nutrients from the sediment. For this reason, this model is particularly well suited to test a bias modeling approach. Our results indicate that calibration without significant amount of bias seems not to be possible for this model. The bias description technique used in this paper seems to address these problems in an adequate way.

4. It is remarkable that the resulting separation of the deviation between model and observations into observation error and bias leads to a significant observation error and bias for phyto- and zooplankton whereas the bias dominates strongly over the observation error for dissolved oxygen, nitrate and phosphate. This result is in qualitative accordance with our prior expectation, but the observation errors are identified to be larger than our priors.

\section{Outlook}

Both, the original bias description technique (Kennedy and O'Hagan 2001, Bayarri et al. 2007. Reichert and Schuwirth 2012) and the simplified, efficient version developed in this paper, are useful techniques to consider the presence of bias in model calibration. The approximate technique has considerable advantages for computationally demanding models where the original technique can not be applied due to long simulation times. Another approach to reduce computation times is emulation. With a dynamic emulator, a statistical description of the behavior of the model, the original bias description technique would be applicable to computationally expensive models as well. Some attempts have been made towards dynamic emulators (Bhattacharya 2007; Conti and O'Hagan 2010, Conti et al. 2009; Liu and West 2009; Reichert et al. 2011). This area is still ongoing research. A hybrid approach, where the inference is done by emulating only the posterior of the model and then propagating this posterior through the original model, is an intermediate stage and subject of future work. It is important to note that the suggested technique is not able to reduce the bias. In addition to the option of considering bias, we would be interested in techniques that support the identification of causes and reduction of bias such as those described in Vrugt et al. (2005), Vrugt and Robinson (2007), Lin and Beck (2007), Reichert and Mieleitner (2009), Bulygina and Gupta (2011) Both the complexity of the bias or structural uncertainty problem and the variation in computational demands of different simulation models require a toolbox of techniques to be available to the modeler. It was the intent of this paper to add a relatively simple and computationally efficient technique to this toolbox. 


\section{Acknowledgments}

We thank Richard Forster and Oliver Köster from WVZ, Barbara Knel, Pius Niederhauser, Irene Purtschert and Peter Spohn from AWEL and Daniel Streit and Christian Leu from BAFU for kindly providing their data on chemistry, biology and physics of Lake Zurich, discharging waste water treatment plants and inflowing rivers. We thank the Swiss National Science Foundation (SNF) for the financial support of the project and three anonymous reviewers for their helpful comments to the manuscript. 


\section{References}

Bates, B. C. and E. P. Campbell (2001). A Markov chain Monte Carlo scheme for parameter estimation and inference in conceptual rainfall-runoff models. Water Resources Research 37(4), 937-947.

Bayarri, M. J., J. O. Berger, R. Paulo, J. Sacks, J. A. Cafeo, J. Cavendish, C.-H. Lin, and J. Tu (2007). A framework for validation of computer models. Technometrics 49(2), 138-154.

Bhattacharya, S. (2007). A simulation approach to Bayesian emulation of complex dynamic computer models. Bayesian Analysis 2(4), 783-816.

Box, G. E. P. and O. R. Cox (1964). An analysis of transformation. J. R. Statist. Soc. B: Statistical Methodology 26(2), 211-252.

Box, G. E. P. and O. R. Cox (1982). An analysis of transformation, revisited, rebutted. Journal of the American Statistical Association 7r(377), 209-210.

Boyle, D. P., H. V. Gupta, and S. Sorooshian (2003). Multicriteria calibration of hydrologic models. In Q. Duan, H. V. Gupta, S. Sorooshian, A. N. Rousseau, and R. Turcotte (Eds.), Calibration of Watershed Models, pp. 185-196. Washington, DC: American Geophysical Union.

Bulygina, N. and H. Gupta (2011). Correcting the mathematical structure of a hydrological model via Bayesian data assimilation. Water Resources Research 47, W05514, doi:10.1029/2010WR009614.

Buser, C. M., H. R. Künsch, D. Lüthi, D. Wild, and Schär (2009). Bayesian multi-model projection of climate: bias assumptions and interannual variability. Climate Dynamics 33, 849-868.

Chen, X. (2011). A three-dimensional hydrodynamic model for shallow waters using unstructured cartesian grids. International Journal for Numerical Methods in Fluids 66, 885-905.

Christensen, J. H., F. Boberg, O. B. Christensen, and P. Lucas-Picher (2008). On the need for bias correction of regional climate change projections if temperature and precipitation. Geophys. Res. Lett. 35, L20709. doi:10.1029/2008GL035694.

Conti, S., J. P. Gosling, J. Oakley, and A. O'Hagan (2009). Gaussian process emulation of dynamic computer codes. Biometrika 96(3), 663-676.

Conti, S. and A. O'Hagan (2010). Bayesian emulation of complex multi-output and dynamic computer models. Journal of Statistical Planning and Inference 140, 640-651.

Craig, P. S., M. Goldstein, J. C. Rougier, and A. H. Seheult (2001). Bayesian forecasting for complex systems using computer simulators. Journal of the American Statistical Association 96, 717-729.

Craig, P. S., M. Goldstein, A. H. Seheult, and J. A. Smith (1996). Bayes linear strategies for 
matching hydrocarbon reservoir history. In J. M. Bernardo, J. O. Berger, and A. P. Dawid (Eds.), Bayesian Statistics 5, pp. 69-95. Oxford: Clarendon Press.

Dee, D. P. and A. M. Da Silva (1998). Data assimilation in the presence of forecast bias. Quarterly Journal of the Royal Meteorological Society 124, 269-295.

Dietzel, A., J. Mieleitner, S. Kardaetz, and P. Reichert (2012). Effects of changing driving forces on water quality and plankton dynamics in three Swiss lakes - Long-term simulations with BELAMO. in preparation.

Duan, Q. Y., V. K. Gupta, and S. Sorooshian (1993). Shuffled complex evolution approach for effective and efficient global minimization. Journal of Optimization Theory and Applications 76(3), $501-521$.

Gelman, A., J. B. Carlin, H. S. Stern, and D. B. Rubin (1995). Bayesian Data Analysis. London: Chapman \& Hall.

Geweke, J. (1989). Bayesian inference in econometric models using Monte Carlo integration. Econometrica 57(6), 1317-1339.

Gupta, H. V., L. A. Bastidas, J. A. Vrugt, and S. Sorooshian (2003). Multiple criteria global optimization for watershed model calibration. In Q. Duan, H. V. Gupta, S. Sorooshian, A. N. Rousseau, and R. Turcotte (Eds.), Calibration of Watershed Models, pp. 125-132. Washington, DC: American Geophysical Union.

Gupta, H. V., S. Sorooshian, T. S. Hogue, and D. P. Boyle (2003). Advances in automatic calibration of watershed models. In Q. Duan, H. V. Gupta, S. Sorooshian, A. N. Rousseau, and R. Turcotte (Eds.), Calibration of Watershed Models, pp. 9-28. Washington, DC: American Geophysical Union.

Gupta, H. V., S. Sorooshian, and P. O. Yapo (1998). Toward improved calibration of hydrologic models: Multiple and noncommensurable measures of information. Water Resources Research 34(4), $751-763$.

Higdon, D., M. Kennedy, J. C. Cavendish, J. A. Cafeo, and R. D. Ryne (2004). Combining field data and computer simulations for calibration and prediction. SIAM Journal on Scientific Computing 26(2), 448-466.

Kennedy, J. and R. C. Eberhart (1995). Particle swarm optimization. In Proc. IEEE int. conf. on neural networks, Volume IV, IEEE sevice center, Piscataway, NJ, USA, pp. 1942-1948.

Kennedy, M. C. and A. O'Hagan (2001). Bayesian calibration of computer models. J. R. Statist. Soc. B: Statistical Methodology 63(3), 425-464. 
Kuczera, G. (1983). Improved parameter inference in catchment models. 1. evaluating parameter uncertainty. Water Resources Research 19(5), 1151-1162.

Lin, Z. and M. B. Beck (2007). On the identification of model structure in hydrological and environmental systems. Water Resources Research 43, W02402, doi:10.1029/2005WR004796.

Liu, F. and M. West (2009). A dynamic modeling strategy for Bayesian computer model emulation. Bayesian Analysis 4(2).

Madsen, H. (2000). Automatic calibration of a conceptual rainfall-runoff model using multiple objectives. Journal of Hydrology 235, 276-288.

Madsen, H., G. Wilson, and H. C. Ammentorp (2002). Comparison of different automated strategies for calibration of rainfall-runoff models. Journal of Hydrology 261, 48-59.

Mieleitner, J. and P. Reichert (2006). Analysis of the transferability of a biogeochemical lake model to lakes of different trophic state. Ecological Modelling 194, 49-61.

Mieleitner, J. and P. Reichert (2008). Modelling functional groups of phytoplankton in three lakes of different trophic state. Ecological Modelling 211, 279-291.

Mooij, W. M., D. Trolle, E. Jeppesen, G. B. Arhonditsis, P. V. Belolipetsky, D. B. R. Chitamwebwa, A. G. Degermendzhy, D. L. DeAngelis, L. N. De Senerpont Domis, A. S. Downing, J. A. Elliott, C. Ruberto Fragoso Jr., U. Gaedke, S. N. Genova, R. D. Gulati, L. Håkanson, D. P. Hamilton, M. R. Hipsey, J. 't Hoen, S. Hülsmann, F. H. Los, V. Makler-Pick, T. Petzoldt, I. G. Prokopkin, K. Rinke, S. A. Schep, K. Tominaga, A. A. Van Dam, E. H. Van Nes, S. A. Wells, and J. H. Janse (2010). Challenges and opportunities for integrating lake ecosystem modelling approaches. Aquatic Ecology 44, 633-667.

Omlin, M., P. Reichert, and R. Forster (2001). Biogeochemical model of Lake Zürich: Model equations and results. Ecological Modelling 141, 77-103.

Reichert, P. (1994). AQUASIM - A tool for simulation and data analysis of aquatic systems. Water Science and Technology 30(2), 21-30.

Reichert, P. (1998). AQUASIM 2.0 - User Manual. Technical report, Swiss Federal Institute for Environmental Science and Technology (EAWAG), Dübendorf, Switzerland.

Reichert, P. (2005). UNCSIM - a computer programme for statistical inference and sensitivity, identifiability, and uncertainty analysis. In Proceedings of the 2005 European Simulation and Modelling Conference (ESM 2005), Oct. 24-26, Porto, Portugal, pp. 51-55. EUROSIS-ETI.

Reichert, P. and J. Mieleitner (2009). Analyzing input and structural uncertainty of nonlinear dynamic models with stochastic, time-dependent parameters. Water Resources Research 45, doi:10.1029/2009WR007814. 
Reichert, P., M. Schervish, and M. J. Small (2002). An efficient sampling technique for Bayesian inference. Technometrics 44(4), 318-327.

Reichert, P. and N. Schuwirth (2012). Linking statistical description of bias to multi-objective model calibration. Water Resources Research. under review.

Reichert, P., G. White, M. J. Bayarri, and E. B. Pitman (2011). Mechanism-based emulation of dynamic simulation models: Concept and application in hydrology. Computational Statistics 8 Data Analysis 55, 1638-1655.

Savić, D. A., J. Bicik, and M. S. Morley (2011). A DSS generator for multiobjective optimisation of spreadsheet-based models. Environmental Modelling and Software 26(5), 551 - 561.

Trelea, I. C. (2003). The particle swarm optimization algorithm: convergence analysis and parameter selection. Information Processing Letters 85, 317-325.

Vrugt, J. A., C. G. H. Diks, H. V. Gupta, W. Bouten, and J. M. Verstraten (2005). Improved treatment of uncertainty in hydrologic modeling: combining the strength of global optimization and data assimilation. Water Resources Research 41(1), W01017, doi:10.1029/2004WR003059.

Vrugt, J. A. and B. A. Robinson (2007). Treatment of uncertainty using ensemble methods: Comparison of sequential data assimilation and bayesian model averaging. Water Resources Research 43, W01411, doi:10.1029/2005WR004838.

Yang, J., P. Reichert, K. C. Abbaspour, and H. Yang (2007). Hydrological modelling of the Chaohe Basin in China: Statistical model formulation and Bayesian inference. Journal of Hydrology 340 , $167-182$.

Yapo, P. O., H. V. Gupta, and S. Sorooshian (1998). Multi-objective global optimization for hydrologic models. Journal of Hydrology 204, 83-97. 